\title{
The Dirichlet problem for minimal surfaces equation and Plateau problem at infinity
}

\author{
Laurent MAZET *
}

\begin{abstract}
In this paper, we shall study the Dirichlet problem for the minimal surfaces equation. We prove some results about the boundary behaviour of a solution of this problem. We describe the behaviour of a non-converging sequence of solutions in term of lines of divergence in the domain. Using this second result, we build some solutions of the Dirichlet problem on unbounded domain. We then give a new proof of the result of C. Cosín and A. Ros concerning the Plateau problem at infinity for horizontal ends.
\end{abstract}

2000 Mathematics Subject Classification. 53A10.

Keywords: Minimal Surface, Dirichlet Problem, Boundary Behaviour.

\section{Introduction}

One classical way to construct minimal surfaces in $\mathbb{R}^{3}$ is to see them as the graph of a function $u$ over a domain $\Omega \subset \mathbb{R}^{2}$ (see for example the paper of H. Karcher [8]). The graph of a function $u$ is a minimal surface if $u$ satisfies the elliptic partial differential equation called the minimal surfaces equation:

$$
\operatorname{div}\left(\frac{\nabla u}{\sqrt{1+|\nabla u|^{2}}}\right)=0
$$

The problem which is associated to this point of view is the Dirichlet problem for the equation (MSE): for a domain $\Omega$ and a function $f$ on $\partial \Omega$, this problem consits in finding a continuous function $u$ on $\bar{\Omega}$ which is a solution of the minimal surfaces equation in $\Omega$ and such that $u=f$ on the

*Laboratoire Emile Picard (UMR 5580), Université Paul Sabatier, 118, route de Narbonne, 31062 Toulouse, France; e-mail mazet@picard.ups-tlse.fr 
boundary of $\Omega$. One of the most general answers to the Dirichlet problem for bounded domain has been given by H. Jenkins and J. Serrin in [7]. They give a nice condition on the domain to solve for any function $f$; moreover, their result allows us to give infinite value for the boundary data $f$. For unbounded domain, the Dirichlet problem is still an open problem. We know that, in the general case, we lose the uniqueness of solution. In this paper, using a new approach, we develop some tools for the study of this problem.

An other interesting and still open problem concerning minimal surfaces is the Plateau problem at infinity which is the following: finding a minimal surface for a given asymptotic behaviour. More precisely, we know that, if a complete minimal surface has finite total curvature and embedded ends, each end of this minimal surface is asymptotic to a plane or to a half-catenoid; besides, we can associate to each end a vector in $\mathbb{R}^{3}$, this vector is called the flux vector of the end. These vectors satisfy the following condition: the sum of the flux vectors over all ends is zero. So the problem is: given a finite number of vectors such that their sum is zero, can we find a minimal surface which has these vectors as flux vectors? Our answer comes from the following idea: seeing a solution of the Plateau problem at infinity as the conjugate surface of a solution of the Dirichlet problem on an unbounded domain.

In [2], C. Cosín and A. Ros give a description of the space of solutions of the Plateau problem at infinity with an asymptotic behaviour which is symmetric with respect to an horizontal plane (i.e. all the flux vectors are horizontal). They also restrict themselves to the case of Alexandrov embedded minimal surfaces; this condition implies that no flux vector is zero and that there is a natural order on the ends of the surface. Since the flux vectors are horizontal and their sum is zero, these vectors draw a polygon in $\mathbb{R}^{2}$. C. Cosín and A. Ros give a necessary and sufficient condition on this polygon to have a solution. See section 4 , for more explanations about their work.

In this paper, we give a more constructive proof of the result of $\mathrm{C}$. Cosín and A. Ros. Our method is based on the Dirichlet problem on an unbounded "domain" $\Omega$. When the polygon given by the flux vectors is convex, $\Omega$ can be defined as the polygonal domain bounded by the flux polygon to which we glue a half-strip on each edge. We note $L_{i}^{+}$and $L_{i+1}^{-}$the two sides of each half-strip $S_{i}$, alternating the sign + and - such that each vertex of the polygon is common to some $L_{i}^{-}$and $L_{i}^{+}$. When the flux polygon is nonconvex and satisfies the condition of C. Cosín and A. Ros, we need to use the concept of mutli-domain for defining $\Omega$ (see Definition 1 for this concept).

Our main result for the Dirichlet problem for this kind of domain $\Omega$ is then (see Theorem [6): 
There exists a solution $u$ of the minimal surfaces equation on $\Omega$ such that $u$ tends to $+\infty$ on $L_{i}^{+}$and $-\infty$ on $L_{i}^{-}$. Besides, the solution is unique up to an additive constant

The function $u$ in this result is build as the limit of solutions of the Dirichlet problem on bounded domain. We describe the possible divergences that can occur for a sequence of solutions of (MSE). In fact, we prove that if the sequence diverges at a point, it must diverge along a line passing by this point. This result is a generalization of the results that $H$. Jenkins and J. Serrin use in [7]. Our result allows us to do the same discussion that H. Jenkins and J. Serrin made in the particular case of monotone sequences of solutions of (MSE); this is our main tools to prove the existence part of Theorem [6.

The solution to the Plateau problem at infinity is then the conjugate surface to the graph of $u$. In order to know the geometry of the conjugate surface along its boundary, we need to understand the behaviour of the graph in the neighborhood of the vertices of $\Omega$ which are the vertices of the polygon. Some results are known for such problem concerning the Dirichlet problem in the convex case. For example, consider $f$ a data on the boundary of a domain $\Omega$, we suppose that $f$ has a finite discontinuity at a point $P$ where the boundary is convex (i.e. we suppose that $f(Q)$ has a limit if we tend to $P$ by the right hand side or by the left hand side and that the difference of these two limits is finite), then we know that the graph of a solution $u$ over $\Omega$ of the Dirichlet problem with $f$ as boundary value, has a vertical segment over $P$ in its boundary, it was proved in [10]. In our case, we can prove that the boundary of the graph is the vertical straight line passing by the vertex ; although the domain is locally an angular sector that not need to be convex and the boundary data takes the values $+\infty$ on one side of the sector and $-\infty$ on the other side.

The paper is organized as follows; in the first section, we define multidomains and extend the result of H. Jenkins and J. Serrin to bounded multi-domains. The multi-domains are necessary to express the condition of C. Cosín and A. Ros. This result will be our first tool in the proof of our main theorem.

The second section is devoted to the proof of our result concerning the boundary behaviour of solutions of the Dirichlet problem.

In section 3. we study the sequences of solutions of (MSE) and define the lines of divergence.

In section 4, we explain the result of C. Cosín and A. Ros, and recall some elements of their proof. In the last section, we give the proof of our 
main result. We then use it to give a new proof of the result of C. Cosín and A. Ros.

Let us fix some notations. In the following, when $u$ is a function on a domain of $\mathbb{R}^{2}$ we shall note $W=\sqrt{1+|\nabla u|^{2}}$. We shall also use the classical following notations for partial derivatives: $p=\frac{\partial u}{\partial x}, q=\frac{\partial u}{\partial y}, r=\frac{\partial^{2} u}{\partial x^{2}}$, $s=\frac{\partial^{2} u}{\partial x \partial y}$ and $t=\frac{\partial^{2} u}{\partial y^{2}}$. Besides, for the graph of $u$, we shall always chose the downward pointing normal to give an orientation to the graph.

\section{The Dirichlet problem on multi-domains}

In this section, we shall give a generalization of the results of H. Jenkins and J. Serrin [7] for the Dirichlet problem on bounded domain. First we have to generalize the notion of domain of $\mathbb{R}^{2}$. Let us consider a pair $(\Omega, \varphi)$ where $\Omega$ is a simply-connected 2-dimensional complete flat manifold with piecewise smooth boundary and $\varphi: \Omega \longrightarrow \mathbb{R}^{2}$ is a local isometry. The map $\varphi$ is called the developing map and the points where the boundary $\partial \Omega$ are not smooth are called vertices.

Definition 1. A pair $(\Omega, \varphi)$, where $\Omega$ is a simply-connected 2-dimensional complete flat manifold with piecewise smooth boundary and $\varphi: \Omega \longrightarrow \mathbb{R}^{2}$ is a local isometry, is a multi-domain if each connected component of the smooth part of $\partial \Omega$ is a convex arc.

If $(\Omega, \varphi)$ is as above and a part of $\partial \Omega$ is linear then we add two vertices at the end points of this linear part and we call this new part an edge.

Let $(\Omega, \varphi)$ be a multi-domain, if $u$ is a smooth function on $\Omega$ we shall call graph of $u$ the surface in $\mathbb{R}^{3}$ given by $\{(\varphi(x), u(x))\}_{x \in \Omega}$. If $u$ is a solution of the minimal surfaces equation (MSE), the graph of $u$ is a minimal surface of $\mathbb{R}^{3}$. The Dirichlet problem on multi-domain consists in the determination of a function $u$ satisfying the equation (MSE) on $\Omega$ and taking on assignated values on the boundary of $\Omega$.

As in the case of a domain in $\mathbb{R}^{2}$, if $u$ is a solution of (MSE) on $\Omega$, we can define a differential form $\mathrm{d} \Psi_{u}$ on $\Omega$ which corresponds to the differential of the third coordinate of the conjugate surface of the graph of $u$. In using the charts given by the developing map $\varphi$, we have $\mathrm{d} \Psi_{u}=\frac{p}{W} \mathrm{~d} y-\frac{q}{W} \mathrm{~d} x \cdot \mathrm{d} \Psi_{u}$ is a closed form by (MSE) and, since $\Omega$ is simply connected, we can define a function $\Psi_{u}$ on $\Omega$ which is 1-Lipschitz continuous, we call this function 
the conjugate function to $u$. One important result concerning $\mathrm{d} \Psi_{u}$ is the following lemma.

Lemma 1. Let $\Omega$ be a domain bounded in part by a straight segment $T$, oriented such that the right hand normal to $T$ is the outer normal to $\Omega$. Let $u$ be a solution of (MSE) in $\Omega$ which assumes the boundary value $+\infty$ on $T$. Then

$$
\int_{T} \mathrm{~d} \Psi_{u}=|T|
$$

This is Lemma 4 in [7]. For other properties of $\Psi_{u}$ and $\mathrm{d} \Psi_{u}$, we refer to this paper.

When $\Omega$ is compact there is a finite number of connected components of the smooth part of $\partial \Omega$; let us call them $C_{1}, \ldots, C_{n}$. When the data on the boundary is bounded, we have this result:

Theorem 1. Let $(\Omega, \varphi)$ be a compact multi-domain with boundary arcs $C_{1}, \ldots$, $C_{n}$ and let $u_{1}, \ldots, u_{n}$ be bounded continuous functions respectively on $C_{1}, \ldots$, $C_{n}$. Then there exists a unique solution $u$ of the minimal surfaces equation on $\Omega$ such that $u_{\mid C_{i}}=u_{i}$.

Proof. The proof of the uniqueness is a particular case of the proof of Theorem 2, so we make it later.

The existence of the solution on multi-domain is due to a Perron process, let us recall some elements of this method. If $v$ is a continuous function on $\Omega$ and $D$ is a disk in $\Omega$, we note by $u_{v, D}$ the solution of (MSE) in $D$ which takes the value $v$ on $\partial D$. We also note $M_{D}[v]$ the continuous function which coincides with $v$ on $\Omega \backslash D$ and $u_{v, D}$ on $D$. Let $u_{1}, \ldots, u_{n}$ be the data on the boundary of $\Omega$; we say that $v$ is a sub-solution of the Dirichlet problem if $v \leq u_{i}$ on $C_{i}$ and $v \leq M_{D}[v]$ for all disks $D$ in $\Omega$. Since the $u_{i}$ are bounded by a constant $M$, the class $\mathcal{F}$ of all sub-solutions is non-empty: the constant function $-M$ is in; besides, each sub-solution $v$ verifies $v \leq M$. So we can define a function $u$ by:

$$
\forall P \in \Omega \quad u(P)=\sup _{v \in \mathcal{F}} v(P)
$$

By standard argument, we can show that $u$ is a solution of (MSE). Since in our definition of multi-domain we suppose that the boundary is locally convex, there exist barrier functions on the boundary (they are constructed in using the Scherk surface). So we can insure that $u$ takes the value $u_{i}$ on $C_{i}$. For more details on Perron process, we can refer to the book of D. Gilbarg and N.S. Trudinger [4] or the one of R. Courant and D. Hilbert [3] which illustrate this method for the classical Laplacian Dirichlet problem, there is 
also the book of J. C. C. Nitsche [10] which studies the case of the minimal surfaces equation.

The work of H. Jenkins and J. Serrin is to allow infinite data on the boundary. By the Straight Line Lemma [7, we know that infinite data can only be allowed on linear parts of the boundary.

Definition 2. Let $(\Omega, \varphi)$ be a multi-domain, a polygonal domain $\mathcal{P}$ of $\Omega$ is a connected compact subset of $\Omega$ such that $(\mathcal{P}, \varphi)$ is a multi-domain, the boundary of $\mathcal{P}$ is only composed of edges and the vertices of $\mathcal{P}$ are drawn from the vertices of $\Omega$.

We want to solve the Dirichlet problem with infinite data so let us call $A_{1}, \ldots, A_{k}$ and $B_{1}, \ldots, B_{l}$ the edges of $\Omega$ such that we assign the value $+\infty$ on $A_{j}$ and $-\infty$ on $B_{j}$. We call $C_{1}, \ldots, C_{n}$ the remaining arcs on which we assign continuous data.

Let $\mathcal{P}$ be a polygonal domain of $\Omega$. We note, respectively, $\alpha$ and $\beta$ the total length of the edges $A_{j}$ and the one of the edges $B_{j}$ which belong to the boundary of $\mathcal{P}$ and we note $\gamma$ the perimeter of $\mathcal{P}$. We then have the following generalization of the result of H. Jenkins and J. Serrin.

Theorem 2. Let $(\Omega, \varphi)$ be a compact multi-domain with the families $\left\{A_{j}\right\}$, $\left\{B_{j}\right\}$ and $\left\{C_{j}\right\}$ as above.

If the familly $\left\{C_{j}\right\}$ is non-empty, then there exists a solution of the minimal surface equation in $\Omega$ which assumes the value $+\infty$ on each $A_{j}$, the value $-\infty$ on each $B_{j}$ and arbitrarily assignated continuous data on each $C_{j}$, if and only if

$$
2 \alpha<\gamma \text { and } 2 \beta<\gamma
$$

for each polygonal domain $\mathcal{P}$ of $\Omega$. If a solution exists, it is unique.

If the familly $\left\{C_{j}\right\}$ is empty, then a solution exists, if and only if

$$
\alpha=\beta
$$

when $\mathcal{P}$ coincides with $\Omega$ and (囝) holds for all other polygonal domains of $\Omega$. In this case, if a solution exists, it is unique up to an additive constant.

Proof. To prove the existence of a solution, we can use the same arguments than H. Jenkins and J. Serrin, so we refer to [7.

The proof of the uniqueness in [7] works also but we give another proof which we can apply in other situations. Let $u_{1}$ and $u_{2}$ be different solutions of (MSE) with the same data on the boundary. In the case where the familly $\left(C_{j}\right)$ is empty, we suppose that $u_{1}-u_{2}$ is not constant; besides, in considering 
$u_{i}-u_{i}(P)$ (where $P \in \Omega$ ), we can assume that $\left\{u_{1}<u_{2}\right\}$ and $\left\{u_{1}>u_{2}\right\}$ are non-empty. In choosing sufficiently small $\varepsilon>0$, we have $\Omega_{\varepsilon}=\left\{u_{1}-\right.$ $\left.u_{2}>\varepsilon\right\} \neq \emptyset$, besides the choise of $\varepsilon$ is such that $\partial \Omega_{\varepsilon}$ is regular. We note $\mathrm{d} \tilde{\Psi}=\mathrm{d} \Psi_{u_{1}}-\mathrm{d} \Psi_{u_{2}}$, since $\mathrm{d} \tilde{\Psi}$ is closed, we have $\int_{\partial \Omega_{\varepsilon}} \mathrm{d} \tilde{\Psi}=0$. Because $u_{1}$ and $u_{2}$ have the same data on the boundary, $\partial \Omega_{\varepsilon}$ does not intersect $\cup_{j} C_{j}$ so $\partial \Omega_{\varepsilon}$ is composed of three parts: one is included in $\cup_{j} A_{j} \bigcup \cup_{j} B_{j}$ on which $\mathrm{d} \tilde{\Psi}=0$ (this is a consequence of Lemma [1), one is included in $\Omega$ and a last part which is composed of some vertices of $\Omega$ but its contribution to the integral is zero. On the second part, let us call it $\widetilde{\partial \Omega_{\varepsilon}}, \nabla u_{1}-\nabla u_{2}$ points in $\Omega_{\varepsilon}$, this part is then oriented by the non-direct normal to $\nabla u_{1}-\nabla u_{2}$ so, by Lemma 2 of $\mathrm{P}$. Collin and R. Krust in [1], $\int_{\widetilde{\partial \Omega_{\varepsilon}}} \mathrm{d} \tilde{\Psi}<0$; this gives us a contradiction.

\section{A result of regularity at the vertices}

The aim of this section is to understand what geometrically happens at a vertex of a multi-domain where two edges $A_{j}$ and $B_{j}$ converge.

For $\beta_{1}<\beta_{2}$ and $R>0$, we consider:

$$
\Omega_{\beta_{1}}^{\beta_{2}}(R)=\left\{(r, \theta) \mid 0 \leq r \leq R, \beta_{1} \leq \theta \leq \beta_{2}\right\}
$$

with the metric $\mathrm{d} s^{2}=\mathrm{d} r^{2}+r^{2} \mathrm{~d} \theta^{2}$ (we identify all the points $(0, \theta)$ and this point will be called the vertex of $\left.\Omega_{\beta_{1}}^{\beta_{2}}(R)\right)$. We define also on $\Omega_{\beta_{1}}^{\beta_{2}}(R)$ the $\operatorname{map} \varphi:(r, \theta) \mapsto(r \cos \theta, r \sin \theta)$. Then $\left(\Omega_{\beta_{1}}^{\beta_{2}}(R), \varphi\right)$ is a multi-domain, it is a description of a neighborhood of a vertex where two edges converge. We call $L(\beta)$ the set of points in $\Omega_{\beta_{1}}^{\beta_{2}}(R)$ such that $\theta=\beta$. We are interested in the geometrical "configuration" of the graph of a solution $u$ of (MSE) such that $u$ tends to $-\infty$ on $L\left(\beta_{2}\right)$ and $+\infty$ on $L\left(\beta_{1}\right)$; such a solution $u$ will be called a solution of the problem $\mathcal{P}$.

The first thing we have to do to understand a solution $u$ of the problem $\mathcal{P}$ is being able to bound the function $u$ on each radius $L(\beta)$. Our arguments are based on the comparison with the Scherk surface.

Let us consider $A B C$ an isosceles triangle $(|A B|=|A C|=R)$, we consider the solution $w$ of the Dirichlet problem on $A B C$ such that $w=0$ on $[A, B]$ and $[A, C]$ and tends to $+\infty$ on $[B, C]$; this function exists by Theorem 2. When $A B C$ is rectangle $w$ is the Scherk surface, after dilatation, $w$ is given by:

$$
w(x, y)=h(x, y)=-\ln \cos x+\ln \cos y
$$

In the general case, the solution $w$ will be called a pseudo Scherk surface. 
We shall use the Scherk surface to control solutions of the problem $\mathcal{P}$. We first consider the case where $A B C$ is rectangle. In fact, a neighborhood of $B$ in $A B C$ can be isometrically parametrized by $\Omega_{-\frac{\pi}{4}}^{0}(R)$ and $h$ is a solution of (MSE) on $\Omega_{-\frac{\pi}{4}}^{0}(R)$ such that $h=0$ on $L(0),+\infty$ on $L\left(-\frac{\pi}{4}\right)$ and some positive function on the third part of the boundary. Since we have an expression for $h$ we can see that $h$ is uniformly bounded on $\Omega_{\alpha}^{0}(R) \subset \Omega_{-\frac{\pi}{4}}^{0}(R)$ for every $-\frac{\pi}{4}<\alpha<0$.

We do not suppose now that $A B C$ is rectangle; but we suppose that the angle at the vertex $A$ is greater than $\frac{\pi}{2}$. In this case we can choose a point $A^{\prime}$ such that $A^{\prime} B C$ is isosceles and rectangle and $A^{\prime} B C$ contains $A B C$. We consider in $A B C$ the pseudo Scherk surface $w$ and $h$ the Scherk surface on $A^{\prime} B C$; since $h$ is positive in $A^{\prime} B C$, we have $h>w$. As above, a neighborhood of $B$ in $A B C$ can be isometrically parametrized by $\Omega_{\beta}^{0}(R)$ with $\beta<0$ and $w$ can be seen as the solution of (MSE) on $\Omega_{\beta}^{0}(R)$ such that $w=0$ on $L(0)$, $+\infty$ on $L(\beta)$ and some positive function on the third part of the boundary. Since $w<h, w$ is uniformly bounded on $\Omega_{\alpha}^{0}(R)$ for every $\beta<\alpha<0$.

By our expression for $h$, there exists $m \in \mathbb{R}$ such that $h \leq m$ on $[A, B]$ and $[A, C]$. This proves that $h-m \leq w$ in $A B C$. Then in our parametrization of a neighborhood of $B$, for every $M \in \mathbb{R}$ there exist $\alpha$ such that $w \geq M$ in $\Omega_{\beta}^{\alpha}(R)$.

Lemma 2. Let $\beta_{1}<\beta_{2}$ and $R>0$. We consider a solution $u$ of the problem $\mathcal{P}$ on $\Omega_{\beta_{1}}^{\beta_{2}}(R)$. Then for every $\beta_{1}<\alpha<\beta_{2}$, there exist $M$ and $M^{\prime}$ in $\mathbb{R}$ such that $u \leq M$ in $\Omega_{\alpha}^{\beta_{2}}\left(\frac{R}{4}\right)$ and $u \geq M^{\prime}$ on $\Omega_{\beta_{1}}^{\alpha}\left(\frac{R}{4}\right)$. For every $M \in \mathbb{R}$, there exist $\alpha$ and $\alpha^{\prime}$ in $] \beta_{1}, \beta_{2}\left[\right.$ such that $u \geq M$ in $\Omega_{\beta_{1}}^{\alpha}\left(\frac{R}{4}\right)$ and $u \leq M$ in $\Omega_{\alpha^{\prime}}^{\beta_{2}}\left(\frac{R}{4}\right)$.

Proof. Let us consider $\alpha_{1}<\alpha_{2}$ and $R^{\prime}>0$. We consider $v$ the solution of the problem $\mathcal{P}$ on $\Omega_{\alpha_{1}}^{\alpha_{2}}\left(R^{\prime}\right)$ such that $v=0$ on the third part of the boundary; $v$ exists because the hypotheses of Theorem 2 are fulfilled. The isometry of $\Omega_{\alpha_{1}}^{\alpha_{2}}\left(R^{\prime}\right) \times \mathbb{R}$ defined by $(r, \theta, z) \mapsto\left(r, \alpha_{1}+\alpha_{2}-\theta,-z\right)$ does not change the boundary data so $v$ is invariant by this isometry because of the uniqueness of such a solution. This proves that $v=0$ on $L\left(\frac{\alpha_{1}+\alpha_{2}}{2}\right)$. Then, by maximum principle, we have $v>0$ between $L\left(\alpha_{1}\right)$ and $L\left(\frac{\alpha_{1}+\alpha_{2}}{2}\right)$ and $v<0$ between $L\left(\frac{\alpha_{1}+\alpha_{2}}{2}\right)$ and $L\left(\alpha_{2}\right)$.

Let us consider $\alpha_{1}<\alpha<\alpha_{2}$. Let us prove that there exists a constant $M$ such that $v \leq M$ in $\Omega_{\alpha}^{\alpha_{2}}\left(\frac{R^{\prime}}{2}\right)$. If $\alpha \geq \frac{\alpha_{1}+\alpha_{2}}{2}, M=0$ works. We note $\widetilde{\alpha}=\frac{\alpha_{1}+\alpha_{2}}{2}$. We suppose $\alpha \leq \widetilde{\alpha}$ then we take a sufficiently big $n$ such that $\frac{\widetilde{\alpha}-\alpha}{n} \leq \frac{\pi}{4}$ and $\frac{\widetilde{\alpha}-\alpha}{n} \leq \alpha-\alpha_{1}$. We note $B$ the vertex of $\Omega_{\alpha_{1}}^{\alpha_{2}}\left(R^{\prime}\right)$. For $k \leq 2 n+1$ we note $\alpha(k)=\widetilde{\alpha}-k \frac{\widetilde{\alpha}-\alpha}{2 n}$ and for $k \leq 2 n-1$ we note $A_{k}$ the points of coordinates $\left(\frac{R^{\prime}}{2}, \alpha(k)\right)$ and $C_{k}$ the point of second coordinate $\alpha(k+2)$ such 
that $A_{k} B C_{k}$ is an isosceles triangle at $A_{k}$ (see Figure 1). We have $v=0$ on $\left[B, A_{0}\right]$ and $v$ is bounded on $\left[A_{0}, C_{0}\right]$, then, by adding a constant, we can put a pseudo Scherk surface above $v$ over $A_{0} B C_{0}$. This proves that $v$ is upperbounded in $\Omega_{\alpha(1)}^{\alpha_{2}}\left(\frac{R^{\prime}}{2}\right)$. Since $v$ is upper-bounded on $\left[B, A_{1}\right]$ and $\left[A_{1}, C_{1}\right]$, we can put a pseudo Scherk surface above $v$ over $A_{1} B C_{1}$ then $v$ is bounded on $\Omega_{\alpha(2)}^{\alpha_{2}}\left(\frac{R^{\prime}}{2}\right)$. We can do this for every $k$ then we obtain that $v$ is uniformly upper-bounded on $\Omega_{\alpha}^{\alpha_{2}}\left(\frac{R^{\prime}}{2}\right)$.

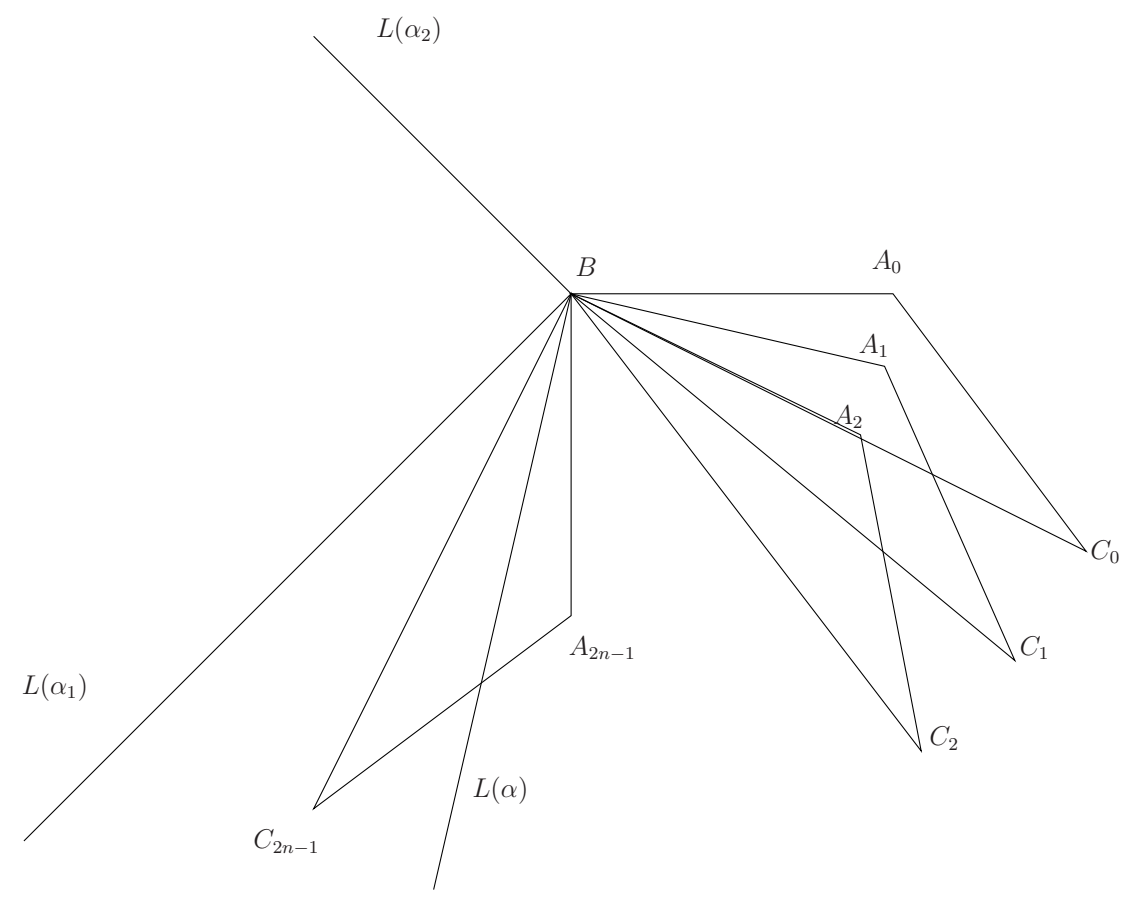

Figure 1:

With the same method, we can prove that there exists $M^{\prime}$ such that $v \geq M^{\prime}$ on $\Omega_{\alpha_{1}}^{\alpha}\left(\frac{R^{\prime}}{2}\right)$.

Let us now consider our original problem. We have $u$ and $\alpha$ and we want to prove the existence of $M$. We consider $\beta_{1}<\alpha^{\prime}<\alpha$, since $u$ tends to $-\infty$ along $L\left(\beta_{2}\right)$, there exists $m$ such that $u \leq m$ at all the points of coordinates $\left(\frac{R}{2}, \theta\right)$ with $\alpha^{\prime} \leq \theta<\beta_{2}$. We consider on $\Omega_{\alpha^{\prime}}^{\beta_{2}}\left(\frac{R}{2}\right)$ the solution $v$ that we have studied above, by maximum principle, we have $u \leq v+m$ on $\Omega_{\alpha^{\prime}}^{\beta_{2}}\left(\frac{R}{2}\right)$. We then have the existence of $M$ because of the result on $v$. We construct $M^{\prime}$ in the same way.

Let us now consider $u$ a solution of $\mathcal{P}$ and $M \in \mathbb{R}$. We consider $\beta_{1}<$ $\beta<\beta_{2}$ such that $\beta-\beta_{1} \leq \frac{\pi}{4}$, we consider the point $A$ of coordinates $\left(\frac{R}{2}, \beta\right)$ and $C$ the point on $L\left(\beta_{1}\right)$ such that $A B C$ is a isosceles triangle (where $B$ 
is the vertex of $\left.\Omega_{\beta_{1}}^{\beta_{2}}\right)$. By what we have just proved, $u$ is lower-bounded on $[B, A]$ and $[A, C]$ then we can put a pseudo Scherk surface under $u$. The existence of $\alpha$ is due to the last remark that we made about pseudo Scherk surfaces

Using this result, we can prove the following geometrical result.

Theorem 3. Let $(\Omega, \varphi)$ be a multi-domain and $P$ a vertex of $\Omega$ such that two edges $L_{1}$ and $L_{2}$ have $P$ as end point ( $L_{1}$ and $L_{2}$ are enumerated with respect to the orientation). Let $u$ be a solution of (MSE) on $\Omega$ such that $u$ tends to $-\infty$ on $L_{1}$ and $+\infty$ on $L_{2}$. We consider $\Psi_{u}$ the conjugate function to $u$ normalized such that $\Psi_{u}(P)=0$. Then, if $\Psi_{u}$ is non-negative in a neighborhood of $P$, the vertical straight line passing through $\varphi(P)$ is the boundary of the graph of $u$ above a neighborhood of $P$.

First, we remark that, if $Q$ is a point on $L_{1}$ or $L_{2}$, then $\Psi_{u}(Q)=|P Q| \geq 0$ by Lemma 1. This proves that, if the angle at $P$ is strictly less than $\pi$, the hypothesis on $\Psi_{u}$ is always verified; so we have the result for a convex corner.

Proof. By a translation and a rotation, we can isometrically parametrized a neighborhood of $P$ by $\Omega_{\beta}^{0}(R)$ for $\beta<0$ and $R$ small enough. Then $u$ can be seen as a solution of the problem $\mathcal{P}$. We suppose that $\Psi_{u} \geq 0$ in $\Omega_{\beta}^{0}(R)$.

\section{First part}

First, we prove that there exists $M_{1} \in \mathbb{R}$ such that $\left.\varphi(P) \times\right]-\infty, M_{1}$ [ is a part of the boundary of the graph. We take $-\frac{\pi}{2}<\alpha<0$, we suppose that $\alpha>\frac{\beta}{2}$. Then $\Omega_{\alpha}^{0}(R) \subset \Omega_{\beta}^{0}(R)$ can be parametrized by euclidean parameters $(x, y)$, in fact $\Omega_{\alpha}^{0}(R)$ is embedded in $\mathbb{R}^{2}$. The idea is to see the part of the graph which is over $\Omega_{\alpha}^{0}(R)$ as a graph over the vertical plane given by the equation $y=0$. Let $R^{\prime}<\frac{R}{2}$, then for all $Q \in \Omega_{\alpha}^{0}\left(R^{\prime}\right)$ the nearest point from $Q$ on $\partial \Omega$ is on $L_{1}$. If we take $R^{\prime}$ small enough and $\alpha$ such that $\tan \alpha>-\frac{1}{8}$, then every point of $\Omega_{\alpha}^{0}\left(R^{\prime}\right)$ verifies the hypothesis of Lemma 1 in [7]. This lemma implies that, at every point of $\Omega_{\alpha}^{0}\left(R^{\prime}\right), q=\frac{\partial u}{\partial y}<0$. Using our euclidean parameters, we note, for $(x, y) \in \Omega_{\alpha}^{0}\left(R^{\prime}\right), \Theta(x, y)=(x, u(x, y))$. We have:

$$
\mathrm{d} \Theta=\left(\begin{array}{cc}
1 & 0 \\
\frac{\partial u}{\partial x} & \frac{\partial u}{\partial y}
\end{array}\right)
$$

Since $q<0$, this proves that $\Theta$ is a local diffeomorphism. Since $u$ strictly decreases when $y$ increases, $\Theta$ is injective. By Lemma 2, we know that there exists $K \in \mathbb{R}$ such that $u \geq K$ on $L(\alpha)$, we put $x_{1}=R^{\prime} \cos \alpha$. We then 
have $] 0, x_{1}[\times]-\infty, K\left[\subset \Theta\left(\Omega_{\alpha}^{0}\left(R^{\prime}\right)\right)\right.$. We note $\chi=\Theta^{-1}$ on $] 0, x_{1}[\times]-\infty, K[$ we then have $y=\chi_{(2)}(x, z)$ on the graph of $u$ (we note $\chi_{(2)}$ the second coordinate function of $\chi$ ) then $\chi_{(2)}$ verifies (MSE). When $x \longrightarrow 0$, we have $y=\chi_{(2)}(x, z) \longrightarrow 0$, it is due to the shape of $\Omega_{\alpha}^{0}\left(R^{\prime}\right)$. From Lemma 2, there exist $\alpha<\alpha^{\prime}<0$ and $r$ such that $\Omega_{\alpha^{\prime}}^{0}(r) \subset \operatorname{im} \chi$. By results of boundary regularity, $\chi_{(2)}$ is regular at the boundary, actually we can extend $\chi_{(2)}$ by making a reflection with respect to the axis $\{x=0, y=0\}$. We now show that a part of this axis is a part of the boundary of the whole graph. By lemma 2. there exists $M^{\prime}$ such that $u \geq M^{\prime}$ in $\Omega_{\beta}^{\alpha^{\prime}}(r)$. We note $M_{1}=M^{\prime}-1<$ $K$; then if a sequence of points of the graph of $u$ over $\Omega_{\beta}^{0}(r)$ tends to a point of $\varphi(P) \times]-\infty, M_{1}$ [, we have $(x, y)$ in im $\chi$ after a certain rank. Then the graph of $u$ over im $\chi$ is a neighborhood of $\varphi(P) \times]-\infty, M_{1}\left[\right.$; as $\chi_{(2)}$ is regular through the boundary, $\varphi(P) \times]-\infty, M_{1}[$ is a part of the boundary.

With the same arguments, we can show that there exists $M_{2}$ such that $\varphi(P) \times] M_{2},+\infty[$ is a part of the boundary.

Second part

The first part proves that outside a compact the graph of $u$ has a good behaviour above the point $\varphi(P)$. Now, we prove that we can extend, by reflection, this compact part through the verical straight line passing by $\varphi(P)$.

From what we have just done, there exist $\beta<\alpha_{2}<\alpha_{1}<0$ such that the graph above $\Omega_{\beta}^{\alpha_{2}}(R)$ and $\Omega_{\alpha_{1}}^{0}(R)$ is regular above $P$. We choose $M_{1}$ and $M_{2}$ as in the first part such that $\left(\varphi(P), M_{1}\right) \in \partial \operatorname{Graph}\left(\left.u\right|_{\Omega_{\alpha_{1}}^{0}(R)}\right)$ and $\left(\varphi(P), M_{2}\right) \in$ $\partial \operatorname{Graph}\left(\left.u\right|_{\Omega_{\beta}^{\alpha_{2}(R)}}\right)$. We shall construct a curve $\Gamma$ as follow: we start from the point $A_{1}=\left(0,0, M_{1}\right)((0,0)=\varphi(P))$, we go down vertically to the point $A_{2}=\left(0,0, M_{1}-1\right)$, then we go to some point $A_{3}=\left(\varepsilon \cos \theta, \varepsilon \sin \theta, M_{1}-1\right)$ in following the level curve $\left\{u=M_{1}-1\right\}$ (we suppose $\varepsilon$ small and $\theta>\alpha_{1}$ ), we then follow the curve

$$
t \mapsto(\varepsilon \cos t, \varepsilon \sin t, u(\varepsilon \cos t, \varepsilon \sin t)),
$$

we let $t$ decreases to some $\theta^{\prime}<\alpha_{2}$ such that $u\left(\varepsilon \cos \theta^{\prime}, \varepsilon \sin \theta^{\prime}\right)=M_{2}+1$ (we note $A_{4}$ the end point), following the level curve $\left\{u=M_{2}+1\right\}$ we go to the point $A_{5}=\left(0,0, M_{2}+1\right)$ and finally we go down to the point $A_{6}=\left(0,0, M_{2}\right)$. We can smooth $\Gamma$ at the points $A_{2}, A_{3}, A_{4}$ and $A_{5}$ such that $A_{2}$ and $A_{5}$ are always in the smooth $\Gamma$ and the new $\Gamma$ is embedded in the graph of $u$. The vertical projection of $\Gamma$ on $\Omega_{\beta}^{0}(R)$ bounds a domain $\widetilde{\Omega}$. We note $\Sigma$ the graph of $u$ above $\widetilde{\Omega}$. Because of our choice of $\Gamma, \Sigma$ extends in a minimal surface $\Sigma^{\prime}$ through $\Gamma$ (The only problem is through $\left[A_{1}, A_{2}\right]$ and $\left[A_{5}, A_{6}\right]$, but the first part says us that we can extend $\Sigma$ through these two 
segments by symmetry). Because $\Sigma$ is a graph, $\Sigma$ is simply connected and its boundary is not empty; the same is true for $\Sigma^{\prime}$. This remark says us that we have conformal parametrization $h_{1}: D \longrightarrow \Sigma^{\prime}$ and $h_{2}: D \longrightarrow \Sigma$ ( $D$ is the unit disk). We put $\widetilde{D}=h_{1}^{-1}(\Sigma)$ and $\widetilde{h}: \widetilde{D} \longrightarrow D$ defined by $\widetilde{h}=h_{2}^{-1} \circ h_{1} ; \widetilde{h}$ is a biholomorphic map. As $h_{1}^{-1}(\Gamma)$ is embedded in $D$, the property of Schönflies is verified at every point ; by the Carathéodory's Theorem, $\widetilde{h}$ extends to an homeomorphism of $\widetilde{D} \cup h_{1}^{-1}(\Gamma)$ into $D \cup V$ where $V$ is part of the boundary of $D$ (for all this argument we refer to appendix A). This proves that we can extend $h_{2}$ in an homeomorphism of $D \cup V$ into $\Sigma \cup \Gamma$. Let us consider $f: D \longrightarrow D^{-}\left(D^{-}=\{(x, y) \in D \mid y<0\}\right)$ a biholomorphic map, then $f$ extends to the boundary. Let us consider the following points on $\Gamma: A_{1.5}=\left(0,0, M_{1}-0.5\right)$ and $A_{5.5}=\left(0,0, M_{2}+0.5\right)$. We note $X=h_{2} \circ H \circ f^{-1}$ where $H$ is a Moebius transformation of the unit disk. We note $B_{i}=X^{-1}\left(A_{i}\right)$ for every $i$. Then, for a suitable choice of $H$, we can have the situation described by Figure 2 .

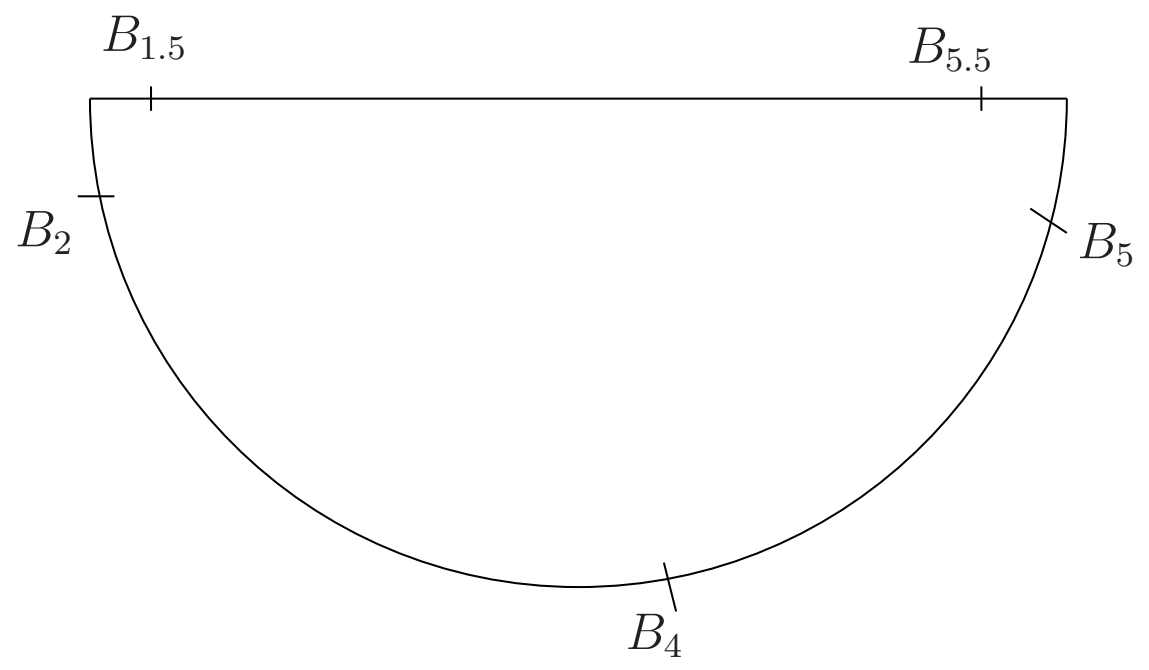

Figure 2:

Let us show that $X$ extends to the whole disk. We shall note $x_{1}, x_{2}$ and $x_{3}$ the three coordinates of $X$, this three functions are harmonic since $\Sigma$ is minimal. First we observe that $x_{1}$ and $x_{2}$ tend to 0 when $z \in D^{-}$ tends to $D^{0}=\{z \in D \mid z \in \mathbb{R}\}$, this is due to the shape of $\widetilde{\Omega}$. Then, by Schwarz reflection principle, $x_{1}$ and $x_{2}$ extend to $D$ in harmonic functions. Let us consider $x_{3}^{*}$ the harmonic conjugate to $x_{3}$ on $D^{-}$, we normalized $x_{3}^{*}$ by $x_{3}^{*}\left(B_{2}\right)=0$. By our choice of normalization, for every $z \in D^{-}$, we have $\Psi_{u}(X(z))=x_{3}^{*}(z)$; this proves that $x_{3}^{*}$ tends to 0 when $z$ tends to $D^{0}$, we can extend $x_{3}^{*}$ by reflection to $D$. By taking the conjugate function, we have 
proved that $x_{3}$ extends to $D$. We then have constructed a minimal immersion $X$ on $D$, then $\Sigma$ extends through $\left[A_{1}, A_{6}\right]$. This extention is given by the reflection with respect to $\left[A_{1}, A_{6}\right]$.

Third part

The last thing we have to show is that the minimal immersion $X$ has no branch point. If it has a branch point then it must be on $D^{0}$, since, on the other part, the surface is a graph and then there is no branch point. Let $z_{0}$ be a branch point, then $\nabla x_{3}^{*}\left(z_{0}\right)=0$. Since $x_{3}^{*}$ is harmonic, its local behaviour is quite similar to the one of $\Re\left(z-z_{0}\right)^{p}$ with $p \geq 2$ (in fact, in some holomorphic chart we have $\left.x_{3}^{*}(z)=\Re\left(z-z_{0}\right)^{p}\right)$. This implies that there exists $z$ in $D^{-}$ such that $x_{3}^{*}(z)<0$, but this contradicts our hypothesis $\Psi_{u} \geq 0$.

We then have proved that there is no branch point so the vertical straight line passing by $\varphi(P)$ is the boundary of the graph.

Remark 1. We can remark that in the first two parts we do not use the hypothesis on $\Psi_{u}$. So in such a situation we can always extend the graph by making a reflection with respect to the vertical axis. But what we obtain is a minimal surface with, may be, a finite number of branch points on the vertical axis.

Remark 2. We can make an other remark. If we consider a vertex $P$, two edges $L_{1}$ and $L_{2}$ having $P$ as end point, and $u$ such that $u$ assumes the data $+\infty$ (or $-\infty$ ) on $L_{1}$ and $L_{2}$, the hypothesis on $\Psi_{u}$ did not have any more sense and the angle at the vertex $P$ must be greater than $\pi$. But we can always apply the two first parts of the proof. The only problem is that we need a result similar to Lemma 2, this is given by Theorem 10.3 in [11. So we can affirm that on the boundary of the graph of $u$ we have a half straight line with a finite number of branch points. Obviously, we must have a branch point at the end point of the half straight line.

\section{Convergence and divergence of sequence of solutions of (MSE)}

In this section we shall explain what we can say when we have a sequence $\left(u_{n}\right)$ of solutions of (MSE) about its convergence: can we make converge a subsequence by some compactness result? What are the different ways of divergence? In [7], it is shown that for a monotone sequence, it appears lines which separate domains of convergence and domains of divergence (this works only for subsequence). We shall show that such lines always appear (Theorem 4). 
First, we have to determine the domain on which we can make converge a sequence, since each surface is a graph, if we want the limit to be a graph, the normal to the surface needs to stay close by the vertical unit vector and then $W_{n}$ have to be bounded. We have then the following lemma.

Lemma 3. Let $\Omega$ be a domain and $\left(u_{n}\right)$ a sequence of solutions of (MSE) on $\Omega$. Let $P \in \Omega$; we suppose that $W_{n}(P)$ is bounded by a constant $M$; then there exists $R>0$ which depends only of $M$ and the distance of $P$ to $\partial \Omega$ such that $W_{n}$ is bounded by $2 M$ on the disk of center $P$ and radius $R$.

Proof. We fix an index $n$. We know (see 9]) that there exists a constant $c$ such that if $u$ is a solution of (MSE) on the disk $\left\{(x, y) \mid x^{2}+y^{2}<R^{2}\right\}$ we have:

$$
r^{2}(0)+2 s^{2}(0)+t^{2}(0) \leq \frac{c}{R^{2}} W^{4}(0)
$$

Let $R$ be such that $2 R=d(P, \partial \Omega)$ then, for all $Q$ in $D(P, R)$, the above equation gives $r^{2}(Q)+2 s^{2}(Q)+t^{2}(Q) \leq \frac{c}{R^{2}} W^{4}(Q)$. We have $\nabla W=$ $\left(\frac{r p+s q}{W}, \frac{s p+t q}{W}\right)$, so, in $D(P, R)$, we have $\|\nabla W\| \leq \widetilde{C} W^{2}$ with $\widetilde{C}$ which depends only of $R$. Let $z$ be the function such that $z(0)=M$ and $z^{\prime}=\widetilde{C} z^{2}$, $z$ is defined on $\left[0, \frac{1}{M \widetilde{C}}[\right.$ by:

$$
\frac{1}{M}-\frac{1}{z}=\widetilde{C} r
$$

Because of our estimate on $\|\nabla W\|$, we have, in polar coordinates, $W(r, \theta) \leq$ $z(r)$. Then $W$ is bounded by $2 M$ on $D\left(P, \min \left(R, \frac{1}{2 M \widetilde{C}}\right)\right)$.

Let $\left(u_{n}\right)$ be a sequence of solutions of (MSE) on a domain $\Omega$. We then define $\mathcal{B}\left(u_{n}\right)$ as the set of the point $Q \in \Omega$ such that $\left(W_{n}(Q)\right)$ is bounded. Lemma 3 says us that $\mathcal{B}\left(u_{n}\right)$ is an open set and that $W_{n}$ is uniformly bounded on each compact inclued in $\mathcal{B}\left(u_{n}\right)$. Then if $D$ is a connected component of $\mathcal{B}\left(u_{n}\right)$ and $P \in D$ there exists an extraction $\theta$ such that $u_{\theta(n)}-u_{\theta(n)}(P)$ converges uniformly on each compact of $D$ to a solution $u$ of (MSE); here, we use some classical compactness results (see 9]). This proves that the divergence of the sequence is due to the behaviour of the sequence over $\Omega \backslash \mathcal{B}\left(u_{n}\right)$.

If $P \in \Omega \backslash \mathcal{B}\left(u_{n}\right)$, there exists a subsequence $u_{n^{\prime}}$ such that $W_{n^{\prime}}(P) \longrightarrow$ $+\infty$. As the normal $N_{n}$ to the graph at $\left(P, u_{n}(P)\right)$ is given by:

$$
N_{n}(P)=\left(\frac{p_{n}}{W_{n}}(P), \frac{q_{n}}{W_{n}}(P),-\frac{1}{W_{n}}(P)\right)
$$


we can suppose that $N_{n^{\prime}}(P)$ converges to an horizontal unit vector. The following proposition describes what locally happens.

Proposition 1. Let $r$ be positive. Let $\left(u_{n}\right)$ be a sequence of solutions of (MSE) on the disk $D(0, r)$. We suppose that $N_{n}(0)$ converges to $(1,0,0)$. Let $\alpha \in] 0,1\left[\right.$, then there exists an extraction $\theta$ such that $N_{\theta(n)}$ converges to $(1,0,0)$ at almost every point of $\{0\} \times[-\alpha r, \alpha r]$.

Proof. Let $n \in \mathbb{N}$, we know (see [1] and [7]) that there exists $\Phi_{n}:(x, y) \mapsto$ $(\xi, \eta)$ with $\Phi_{n}(0,0)=(0,0)$ and:

$$
\begin{aligned}
& \mathrm{d} \xi=\left(1+\frac{1+p_{n}^{2}}{W_{n}}\right) \mathrm{d} x+\frac{p_{n} q_{n}}{W_{n}} \mathrm{~d} y \\
& \mathrm{~d} \eta=\frac{p_{n} q_{n}}{W_{n}} \mathrm{~d} x+\left(1+\frac{1+q_{n}^{2}}{W_{m}}\right) \mathrm{d} y
\end{aligned}
$$

We know that $\Phi_{n}$ increases distance so it is bijective on its image. This image contains the disk of center $(0,0)$ and radius $r$. Besides, we know that $(\xi, \eta)$ are conformal parameters for the graph of $u_{n}$. On the $\xi \eta$ disk $D(0, r)$ we then have the Gauss map $g_{n}(\xi+i \eta)$ which corresponds to the stereographic projection of $N_{n} ; g_{n}$ is holomorphic. We note $z_{n}=g_{n}(0)$, by hypothesis we have $z_{n} \longrightarrow 1$. We note $z=\xi+i \eta$; by our choice of normal $g_{n}: D(0, r) \longrightarrow D(0,1)$, then there exists $h_{n}: D(0, r) \longrightarrow D(0,1)$ holomorphic with $h_{n}(0)=0$ such that:

$$
g_{n}(z)=\frac{h_{n}(z)+z_{n}}{1+\overline{z_{n}} h_{n}(z)}
$$

Since $z_{n} \longrightarrow 1$, the sequence of holomorphic functions $z \longmapsto \frac{z+z_{n}}{1+\overline{z_{n}} z}$ converges simply to 1 on $D(0,1)$ and uniformly on the disk $D(0, \alpha)$ for all $\alpha<$ 1. But by Schwarz Lemma, we have, for all $n \in \mathbb{N}, h_{n}(D(0, \alpha r)) \subset D(0, \alpha)$, we then have uniform convergence of $g_{n}$ to 1 on $D(0, \alpha r)$. In using (15), this proves that for every $\varepsilon$, if $n$ is big enough, we can say that: $\frac{p_{n}}{W_{n}} \geq 1-\varepsilon$ and $\frac{\left|q_{n}\right|}{W_{n}} \leq \varepsilon$ in $\Phi_{n}^{-1}(D(0, \alpha r))$. So to conclude, we need to understand the shape of $\Phi_{n}^{-1}(D(0, \alpha r))$; we shall see that these sets are concentrating along the segment $\{0\} \times[-\alpha r, \alpha r]$.

For all $n \in \mathbb{N}$, we consider, in the $\xi \eta$ disk, the path $\left.\gamma_{n}:\right]-r, r[\longrightarrow D(0, r)$ defined by $\gamma_{n}(0)=0$ and $\gamma_{n}^{\prime}=\frac{\nabla y_{n}}{\left\|\nabla y_{n}\right\|}$ where $y_{n}$ is the second coordinate of $\Phi_{n}^{-1}$. We have (see [11]):

$$
\nabla y_{n}=\left(-\frac{p_{n} q_{n}}{J_{n} W_{n}}, \frac{W_{n}+1+p_{n}^{2}}{J_{n} W_{n}}\right)
$$


where $J_{n}=\operatorname{det}\left(\mathrm{d} \Phi_{n}\right)=W_{n}+2+\frac{1}{W_{n}}$. Because (5), $\nabla y_{n}$ converges uniformly to $(0,1)$ on the disk $D(0, \alpha r)$ for all $\alpha<1$.

Let $\alpha \in] 0,1\left[\right.$, we note $A_{\alpha}$ and $B_{\alpha}$ the points in the $x y$-disk $D(0, r)$ of respective coordinates $(0,-\alpha r)$ and $(0, \alpha r)$. In the following, we prove that $\int_{\left[A_{\alpha}, B_{\alpha}\right]} \mathrm{d} \Psi_{u_{n}} \longrightarrow 2 \alpha r$.

Let $\widetilde{\alpha}>\alpha$, then for $n$ big enough, we have $\left\|\nabla y_{n}\right\|>\frac{\alpha}{\widetilde{\alpha}}$ in $D(0, \widetilde{\alpha} r)$. Because, for $t \in[-\widetilde{\alpha} r, \widetilde{\alpha} r], \gamma_{n}(t) \in D(0, \widetilde{\alpha} r)$, there exists $\widetilde{\alpha} r \leq t_{0}^{n}<t_{1}^{n} \leq \widetilde{\alpha} r$ such that $y_{n}\left(\gamma_{n}\left(t_{0}^{n}\right)\right)=-\alpha r$ and $y_{n}\left(\gamma_{n}\left(t_{1}^{n}\right)\right)=\alpha r$. Along $\left[t_{0}^{n}, t_{1}^{n}\right], y_{n} \circ \gamma_{n}$ increases strictly from $-\alpha r$ to $\alpha r$, then the path $\Gamma_{n}=\Phi_{n}^{-1} \circ \gamma_{n}$ on $\left[t_{0}^{n}, t_{1}^{n}\right]$ can be parametrized by $y \in[-\alpha r, \alpha r]$ : we have a function $f_{n}$ on $[-\alpha r, \alpha r]$ such that for $t \in\left[t_{0}^{n}, t_{1}^{n}\right] x_{n}\left(\gamma_{n}(t)\right)=f_{n}\left(y_{n}\left(\gamma_{n}(t)\right)\right)$. We have:

$$
\begin{aligned}
\left|x_{n} \circ \gamma_{n}(t)\right| & \leq\left|\int_{0}^{t}\right|\left(x_{n} \circ \gamma_{n}\right)^{\prime}(u)|\mathrm{d} u| \\
& \leq\left|\int_{0}^{t}\right|\left|\nabla x_{n}\right|\left|\left(\gamma_{n}(u)\right) \mathrm{d} u\right|=\left|\int_{0}^{t}\left(\frac{1+q_{n}^{2}}{\left(1+W_{n}\right)^{2}}\right)^{\frac{1}{2}}\left(\Gamma_{n}(u)\right) \mathrm{d} u\right|
\end{aligned}
$$

(for the last equality see [11]), then for $n$ big enough $\left|x_{n} \circ \gamma_{n}\right|$ on $\left[t_{0}^{n}, t_{1}^{n}\right]$ can be bounded by a constant uniformly small; this is due to the fact that $\frac{\left|q_{n}\right|}{W_{n}} \leq \varepsilon$ for big $n$. We then have proved that the path $\Gamma_{n}$ is close by the segment $\left[A_{\alpha}, B_{\alpha}\right]$ for big $n$.

Let us now calculate $\int_{\Gamma_{n}} \mathrm{~d} \Psi_{u_{n}}$. We have:

$$
\int_{\Gamma_{n}} \frac{p_{n}}{W_{n}} \mathrm{~d} y-\frac{q_{n}}{W_{n}} \mathrm{~d} x=\int_{-\alpha r}^{\alpha r}\left(\frac{p_{n}}{W_{n}}\left(f_{n}(y), y\right)-\frac{q_{n}}{W_{n}}\left(f_{n}(y), y\right) f_{n}^{\prime}(y)\right) \mathrm{d} y
$$

We have $\left|f_{n}^{\prime}\left(y_{n}\left(\gamma_{n}(t)\right)\right)\right| \leq \frac{\left\|\nabla x_{n}\right\|}{\left\|\nabla y_{n}\right\|}\left(\gamma_{n}(t)\right) \longrightarrow 0$, the convergence is uniform so $f_{n}^{\prime}$ tends uniformly to 0 on $[-\alpha r, \alpha r]$. This proves that $\int_{\Gamma_{n}} \mathrm{~d} \Psi_{u_{n}} \longrightarrow 2 \alpha r$

Let us consider the path $\widetilde{\Gamma_{n}}$ which consists on the segment $\left[A_{\alpha}, B_{\alpha}\right]$, then the segment $\left[B_{\alpha}, \Gamma\left(t_{1}^{n}\right)\right]$, then $-\Gamma_{n}$, then, finally, the segment $\left[\Gamma_{n}\left(t_{0}^{n}\right), A_{\alpha}\right]$. Let $\varepsilon>0$, for $n$ big enough, we can suppose that $\int_{\Gamma_{n}} \mathrm{~d} \Psi_{u_{n}} \geq 2 \alpha r-\varepsilon$ and $\left(\frac{1+q_{n}^{2}}{\left(1+W_{n}\right)^{2}}\right)^{\frac{1}{2}} \leq \varepsilon$ on $\Phi_{n}^{-1}(D(0, \widetilde{\alpha} r))$. As $\mathrm{d} \Psi_{u_{n}}$ is closed $\int_{\widetilde{\Gamma_{n}}} \mathrm{~d} \Psi_{u_{n}}=0$; we 
then have:

$$
\begin{aligned}
2 \alpha r \geq \int_{\left[A_{\alpha}, B_{\alpha}\right]} \mathrm{d} \Psi_{u_{n}} & =-\int_{\left[B_{\alpha}, \Gamma\left(t_{1}^{n}\right)\right]} \mathrm{d} \Psi_{u_{n}}-\int_{-\Gamma_{n}} \mathrm{~d} \Psi_{u_{n}}-\int_{\left[\Gamma_{n}\left(t_{0}^{n}\right), A_{\alpha}\right]} \mathrm{d} \Psi_{u_{n}} \\
& \geq \int_{\Gamma_{n}} \mathrm{~d} \Psi_{u_{n}}-\left|x_{n}\left(\gamma\left(t_{1}^{n}\right)\right)\right|-\left|x_{n}\left(\gamma\left(t_{0}^{n}\right)\right)\right| \\
& \geq 2 \alpha r-\varepsilon-\varepsilon\left|t_{1}^{n}\right|-\varepsilon\left|t_{0}^{n}\right| \\
& \geq 2 \alpha r-\varepsilon(1+2 \widetilde{\alpha} r)
\end{aligned}
$$

This proves that $\int_{\left[A_{\alpha}, B_{\alpha}\right]} \mathrm{d} \Psi_{u_{n}} \longrightarrow 2 \alpha r$ as $n$ tends to $+\infty$. We have $\int_{\left[A_{\alpha}, B_{\alpha}\right]} \mathrm{d} \Psi_{u_{n}}=\int_{\left[A_{\alpha}, B_{\alpha}\right]} \frac{p_{n}}{W_{n}} \mathrm{~d} y$. Because $\frac{p_{n}}{W_{n}} \leq 1$, the preceding equalities prove that $\frac{p_{n}}{W_{n}}$ converges to 1 in $\mathrm{七}^{1}\left(\left[A_{\alpha}, B_{\alpha}\right]\right)$. Then there exists an extraction $\theta$ such that $\frac{\stackrel{p}{\theta(n)}_{W_{\theta(n)}}}{W_{\theta}}$ converges simply to 1 at almost every point in $\left[A_{\alpha}, B_{\alpha}\right]$; thus the proposition is proved.

This proposition gives us a local result and we have the following global result

Theorem 4. Let $(\Omega, \varphi)$ be a multi-domain. Let $\left(u_{n}\right)$ be a sequence of solutions of (MSE) on $\Omega$. Let $P \in \Omega$ and $N$ a unit tangent vector at $P$, we call $D$ the geodesic of $\Omega$ passing at $P$ and normal to $N$. If the sequence $\left(N_{n}(P)\right)$ converges to $N$, then $N_{n}(Q)$ converges to $N$ at every point of $D$.

As $\Omega$ is locally isometric to $\mathbb{R}^{2}$, we have allowed us to call $N$ the parallel transport of $N$ along $D$.

Proof. We first get a parametrization of $D$ by arc-length with $P$ as originpoint; then $D$ is parametrized by $] a, b[]-,\infty, b[] a,,+\infty[$ or $]-\infty,+\infty[$, we shall suppose that we are in the case $] a,+\infty[$ (the other cases are similar). We then consider the set $\mathcal{F}$ of $t \in \mathbb{R}_{+}^{*}$ such, if $\theta_{1}$ is an extraction, there exists a sub-extraction $\theta_{2}$ such that $N_{\theta_{2}(n)}(Q)$ converge to $N$ at almost every $Q$ of the part of $D$ parametrized by $] a+\frac{|a|}{t+1}, t[(a<0)$. Let us prove that $\mathcal{F}=\mathbb{R}_{+}^{*}$. First, we observe that, if $t_{1} \in \mathcal{F}$ and $t_{2}<t_{1}, t_{2} \in \mathcal{F}$. From Proposition 1. there exists $t>0$ such that $t \in \mathcal{F}$. Let $t_{0}=\sup \mathcal{F}$ and suppose that $t_{0}<+\infty$. We consider $P_{1}$ and $P_{2}$ the points on $D$ parametrized by $a+\frac{|a|}{1+t_{0}}$ and $t_{0}$. We choose $R>0$ such that $D\left(P_{i}, R\right) \subset \Omega$ for $i=1,2$. 
Let $\theta_{1}$ be an extraction. Since $t_{0}=\sup \mathcal{F}$, there exist $Q_{1} \in D\left(P_{1}, \frac{R}{3}\right) \cap D$, $Q_{2} \in D\left(P_{2}, \frac{R}{3}\right) \cap D$ and a sub-extraction $\theta_{2}$ such that $N_{\theta_{2}(n)}$ converges to $N$ at $Q_{1}$ and $Q_{2}$. We have $D\left(Q_{i}, \frac{2 R}{3}\right) \subset \Omega$, we then apply Proposition 1 to points $Q_{1}$ and $Q_{2}$ with $\alpha=\frac{3}{4}$. We then have a sub-extraction $\theta_{3}$ such that $N_{\theta_{3}}$ converges to $N$ at almost every point of $D\left(Q_{i}, \frac{R}{2}\right) \cap D$ for $i=1,2$; this proves that $t_{0}$ is not $\sup \mathcal{F}$, because $N_{\theta_{3}}$ converges to $N$ at almost every point of the part of $D$ parametrized by an open interval that contains the segment $\left[a+\frac{|a|}{t_{0}+1}, t_{0}\right]$.

By a standard diagonal process, we can then construct an extraction $\theta$ such that $N_{\theta(n)}$ converges to $N$ at almost every point of $D$. Let $Q$ be in $D$ and we consider $N^{\prime}$ a cluster point of the sequence $N_{\theta(n)}(Q)$, if the third coordinate of $N^{\prime}$ is negative then there exists a sub-extraction $\theta^{\prime}$ such that $W_{\theta^{\prime}(n)}(Q)$ is bounded but this is impossible since, by Lemma 3, $W_{\theta^{\prime}(n)}$ would be bounded in a neighborhood of $Q$ and $W_{\theta(n)}$ diverges at almost every point of $D$. Thus the third coordinate of $N^{\prime}$ is 0 ; if $N^{\prime} \neq N$, applying what we have already proved, it appears a second geodesic $D^{\prime}$ passing by $Q$ normal to $N^{\prime}$ and an extraction $\theta^{\prime}$ such that $N_{\theta^{\prime}(n)}$ converges to $N^{\prime}$ at almost every point of $D^{\prime}$. We parametrized $D$ and $D^{\prime}$ by arc-length in using the orientation given by the direct normal to $N$ and $N^{\prime}$, we choose $Q$ as origin point. Let $\varepsilon>0$; we note $A$ the point on $D$ of coordinate $-\varepsilon$ and $B$ the point on $D^{\prime}$ of coordinate $\varepsilon$. For $\varepsilon$ small enough, the triangle $A Q B$ is in $\Omega$ and then $\int_{A Q B} \mathrm{~d} \Psi_{u_{\theta^{\prime}(n)}}=0$. We let $n$ tends to $+\infty$ and then obtain $|A C|+|B C| \leq|A B|$ which contradicts the triangle inequality. We then have proved that $N_{\theta(n)}$ converges to $N$ at every point of $D$. We then have proved that for every extraction $\theta$ we can construct a sub-extraction $\theta^{\prime}$ such that $N_{\theta^{\prime}(n)}$ converges to $N$ at every point of $D$

To finish the proof, we take a point $Q$ in $D$ and suppose that $N_{\alpha(n)}(Q)$ converge to $N^{\prime}$ with $\alpha$ an extraction. Since $N_{\alpha(n)}(P) \longrightarrow N$, there exists a sub-extraction $\alpha^{\prime}$ such that $N_{\alpha^{\prime}(n)}$ converges to $N$ at every point of $D$, in particular at $Q$, then $N=N^{\prime}$.

Remark 3. We then understand the structure of the complementary of $\mathcal{B}\left(u_{n}\right)$, it is a set of geodesics of $\Omega$; one of these geodesics will be called a line of divergence. Then when we have a sequence of solutions of (MSE), the problem of the convergence of the sequence is linked to the understanding of: which lines of divergence are possible? The answer is, in general, given by the behaviour at the boundary. The existence of such lines will permit us to use arguments that are similar to the ones used by H. Jenkins and J. Serrin in [7]. 
The behaviour of the normal along a line of divergence says us that the limit of $\int_{T} \mathrm{~d} \Psi_{u_{n}}$, where $T$ is a segment of a line of divergence with the orientation given by the limit normal, is $|T|$. In the following, we shall draw this limit normal on the figures to explain our arguments.

\section{The Plateau problem at infinity}

In this section, we explain the problem studied by C. Cosín and A. Ros in [2] and give the main results of their paper with some elements of proofs.

Let $\mathrm{M}$ be a complete minimal surface with finite total curvature in $\mathbb{R}^{3}$; we know that $M$ is isometric to a compact Riemann surface minus a finite number of points (we can refer to [11]). $M$ then has a finite number of annular ends; when these ends are embedded they are asymptotic either to a half-catenoid or to a plane. A properly immersed minimal surface with $r$ embedded ends will be called a $r$-noid. We can associate to each end a vector which caracterizes the direction and the growth of the asymptotic half-catenoid (when the end is asymptotic to a plane this vector is zero); this vector is called the flux of the end (for a precise definition of the flux see [6]). If $v_{1}, \ldots, v_{r}$ are the fluxes at each end, we have the following balancing condition:

$$
v_{1}+\cdots+v_{r}=0
$$

This condition tells us that the total flux of the system vanishes. If $v_{1}, \ldots, v_{r}$ are vectors in $\mathbb{R}^{3}$ such that (13) is verified and $g$ is a non-negative integer, the Plateau problem at infinity for these data is to find an $r$-noid of genus $g$ which has $v_{1}, \ldots, v_{r}$ as fluxes at its ends.

Let $\psi: M \longrightarrow \mathbb{R}^{3}$ be an $r$-noid. $M$ is conformally equivalent to a compact surface $\bar{M}$ minus a finite number of points $p_{1}, \ldots, p_{r}$. We will say that $M$ is Alexandrov-embedded if $\bar{M}$ bounds a compact 3-manifold $\bar{\Omega}$ and the immersion $\psi$ extends to a proper local diffeomorphism $f: \bar{\Omega} \backslash\left\{p_{1}, \ldots, p_{r}\right\} \longrightarrow \mathbb{R}^{3}$. An Alexandrov-embedded surface has a canonical orientation; we choose the Gauss map to be the outward pointing normal. An Alexandrov-embedded $r$ noid can not have a planar end (see [2]). We call $\mathcal{M}_{r}$ the space of Alexandrovembedded $r$-noids of genus 0 and $r$ horizontal catenoidal ends. We identify two elements in $\mathcal{M}_{r}$ which differ by a translation. In [2], C. Cosín and A. Ros give a nice description of the space $\mathcal{M}_{r}$.

Let $\psi: M \longrightarrow \mathbb{R}^{3}$ be a nonflat immersion of a connected orientable surface $M$ and $\Pi$ be a plane in $\mathbb{R}^{3}$, normalized to be $\left\{x_{3}=0\right\}$. We note by $S$ the Euclidiean symmetry with respect to $\Pi$ and consider the subsets: 


$$
\begin{aligned}
& M^{+}=\left\{p \in M \mid x_{3}(p)>0\right\} \\
& M^{-}=\left\{p \in M \mid x_{3}(p)<0\right\} \\
& M^{0}=\left\{p \in M \mid x_{3}(p)=0\right\}
\end{aligned}
$$

With these notation we have:

Definition 3. We shall say that $M$ is strongly symmetric with respect to $\Pi$ if

- There exists an isometric involution $s: M \longrightarrow M$ such that $\psi \circ s=$ $S \circ \psi$.

- $\{p \in M \mid s(p)=p\}=M^{0}$.

- The third coordinate $N_{3}$ of the Gauss map of $M$ takes positive (resp. negative) values on $M^{+}$(resp. $M^{-}$).

In 2], C. Cosín and A. Ros prove

Proposition 2. Let $M$ be an r-noid with horizontal ends. Then $M$ is strongly symmetric with respect to an horizontal plane if and only if $M$ is Alexandrov-embedded.

We then use the notion of strong symmetry to study $\mathcal{M}_{r}$; in the following, we always suppose that the plane of strong symmetry is the plane $\left\{x_{3}=0\right\}$. If $M \in \mathcal{M}_{r}, s$ extends to $\bar{M}=\overline{\mathbb{C}}$, the involution $s$ is $z \mapsto \frac{1}{\bar{z}}$ and the points $p_{1}, \ldots, p_{r}$ are in $\{z \in \mathbb{C}|| z \mid=1\}$. We then have an order on $\left\{p_{1}, \ldots, p_{r}\right\}$, let us suppose that $p_{1}, \ldots, p_{r}$ are put in this order. Let $v_{1}, \ldots, v_{r}$ be vectors in $\mathbb{R}^{3}$ such that $2 v_{i}$ is the flux at the end $p_{i}$. We have $v_{1}+\cdots+v_{r}=0$, so if we draw the vectors consecutively in the plane, we get a piece-wise linear closed curve: a polygon. We note $F(M)$ this polygon.

We say that a polygon $V$ bounds an immersed polygonal disk if there exists a compact multi-domain $(\mathcal{P}, \varphi)$ such that $\partial \mathcal{P}$ is only composed of edges and $\varphi(\partial \mathcal{P})=V$.

Then the most important result in [2] is

Theorem 5. Let $v_{1}, \ldots, v_{r}$ be horizontal vectors such that $v_{1}+\cdots+v_{r}=0$ and $V$ the associated polygon, then there exists $M \in \mathcal{M}_{r}$ such that $F(M)=V$ if, and only if, $V$ bounds an immersed polygonal disk 
Besides, we have as much $M \in \mathcal{M}_{r}$ such that $F(M)=V$ as immersed polygonal disks bounded by $V$. Let $V$ be a polygon and $(\mathcal{P}, \varphi)$ a compact multi-domain such that $\varphi(\mathcal{P})$ is an immersed polygonal disk bounded by $V$. Let $P_{1}, \ldots, P_{r}$ be the vertices of $\mathcal{P}$ which are identified with the ones of $V$; we put $P_{1}=P_{r+1}$. Let $i \in\{1, \ldots, r\}$, we can glue to $\mathcal{P}$ along $\left[P_{i}, P_{i+1}\right]$ a half-strip $S_{i}$ isometric to $\left[P_{i}, P_{i+1}\right] \times \mathbb{R}_{+}$. We get a multi-domain which we call $\Omega(\mathcal{P})$; the boundary of $\Omega(\mathcal{P})$ is composed of $2 r$ half straight-lines, we call $L_{i}^{-}$(resp. $L_{i}^{+}$) the half line in the boundary which has $P_{i}$ as end point and is in $S_{i-1}$ (resp. $S_{i}$ ).

Let $M$ be in $\mathcal{M}_{r}$, we consider $\left(M^{+}\right)^{*}$ the conjugate surface to $M^{+}$for the outward pointing normal. In [2], the authors prove that it exists $(\mathcal{P}, \varphi)$ a multi-domain bounded by $F(M)$ such that $\left(M^{+}\right)^{*}$ is a graph over the multidomain $\Omega(\mathcal{P})$; the normal to the graph is the upward pointing normal by Definition 3 . If $u$ is the function on $\Omega(\mathcal{P})$ that gives $\left(M^{+}\right)^{*}$, they prove that $u$ tends to $+\infty$ (resp. $-\infty)$ on $L_{i}^{+}$(resp. $L_{i}^{-}$). C. Cosín and A. Ros use these arguments to prove that if the Plateau problem at infinity has a solution the flux polygon $F(M)$ bounds an immersed polygonal disk. For the other implication, they prove that the map $F: M \mapsto F(M)$ is a covering map to conclude, they use a compactness argument and prove that the space $\mathcal{M}_{r}$ has a smooth structure.

In the next section, we shall solve on $\Omega(\mathcal{P})$ the Dirichlet problem for the boundary data $+\infty$ on $L_{i}^{+}$and $-\infty$ on $L_{i}^{-}$. We shall then take the conjugate of the graph of the solution for the downward pointing normal and so build the solution to the Plateau problem at infinity. The change of orientation makes that we get the surface we want.

\section{The construction of a solution of the Plateau problem at infinity}

The first part of this section will be devoted to the proof of our main result.

Theorem 6. Let $V$ be a polygon wich bounds an immersed polygonal disk $(\mathcal{P}, \varphi)$, we define $\Omega(\mathcal{P})$ as in the preceding section. Then there exists a solution $u$ of (MSE) on $\Omega(\mathcal{P})$ such that $u$ tends to $+\infty$ on $L_{i}^{+}$and $-\infty$ on $L_{i}^{-}$. Besides, the solution is unique up to an additive constant.

Let us first consider $u$ a solution of (MSE) on the half-strip $[0, a] \times \mathbb{R}_{+}$ such that $u$ tends to $-\infty$ on $\{a\} \times \mathbb{R}_{+}^{*}$ and $+\infty$ on $\{0\} \times \mathbb{R}_{+}^{*}$. This situation describes the behaviour in the $r$ half-strips $S_{i}$. Then by Lemma 1 in [7] we have: 


$$
\begin{gathered}
\frac{|q|}{W}(x, y) \geq 1-\frac{a^{2}}{x^{2}} \\
\frac{|p|}{W}(x, y) \leq \sqrt{2} \frac{a}{x}
\end{gathered}
$$

when $x \geq 4 a$. We consider now the general problem.

We begin in proving the uniqueness part of Theorem 6. Let $u_{1}$ and $u_{2}$ be two different solutions of the problem (i.e. $u_{1}-u_{2}$ is non-constant). As in the proof of Theorem 2] we can suppose that $\left\{u_{1}>u_{2}\right\}$ and $\left\{u_{1}<u_{2}\right\}$ are non-empty. Let us call $\Omega_{l}$ the subset of $\Omega(\mathcal{P})$ which is the union of $\mathcal{P}$ and the set of points in each $S_{i}$ that are at a distance less than $l$ from $\left[P_{i}, P_{i+1}\right]$; we define $\Omega_{l}^{+}=\Omega_{l} \cap\left\{u_{1}>u_{2}\right\}$. Let us consider:

$$
I=\int_{\partial \Omega_{l}^{+}} \mathrm{d} \tilde{\Psi}
$$

where $\mathrm{d} \tilde{\Psi}=\mathrm{d} \Psi_{u_{1}}-\mathrm{d} \Psi_{u_{2}}$. Since $\mathrm{d} \tilde{\Psi}$ is closed, we have $I=0 . \quad \partial \Omega_{l}^{+}$is composed of a part which is included on $\left.\left(\cup_{i} L_{i}^{+}\right]\right) \cup\left(\cup_{i} L_{i}^{-}\right)$where $\mathrm{d} \tilde{\Psi}=0$, a part included in the interior of $\Omega(\mathcal{P})$, noted $\Gamma_{l}$, and a part in $I_{i, l}$ which is the part in $S_{i}$ parametrized by $\left[P_{i}, P_{i+1}\right] \times\{l\}$. On the part included in $I_{i, l}$ if $l$ is big enough the integral of $\mathrm{d} \tilde{\Psi}$ is less than $2 \sqrt{2} \frac{\left|P_{i} P_{i+1}\right|^{2}}{l}$ by (15). We then have:

$$
0=I \leq \int_{\Gamma_{l}} \mathrm{~d} \tilde{\Psi}+\sum_{i=1}^{r} 2 \sqrt{2} \frac{\left|P_{i} P_{i+1}\right|^{2}}{l}
$$

By Lemma 2 in [1], $\int_{\Gamma_{l}} \mathrm{~d} \tilde{\Psi}$ is negative and decreases as $l$ increases. Because $\sum_{i=1}^{r} \sqrt{2} \frac{\left|P_{i} P_{i+1}\right|^{2}}{l} \underset{l \rightarrow+\infty}{\longrightarrow} 0$, we get a contradiction. This proves that, if $u_{1}$ and $u_{2}$ are two solutions of our Dirichlet problem, there exists $c \in \mathbb{R}$ such that $u_{1}=u_{2}+c$.

We now prove the existence of the solution. We fix a point $P_{0}$ in $\mathcal{P}$. Let us consider in $S_{i}$ the point $Q_{i}^{k}$ which is the middle point of $I_{i, k}$, we then define $\Omega_{k}$ to be the compact subdomain of $\Omega(\mathcal{P})$ bounded by the segments $\left[P_{i}, Q_{i}^{k}\right]$ and $\left[Q_{i}^{k}, P_{i+1}\right]$. Let $\mathcal{G}_{i}^{k}$ be the set of the points $Q$ in $\Omega_{k}$ such that $d\left(Q, Q_{i}^{k}\right)<d\left(P_{i}, Q_{i}^{k}\right)$; if $k$ is big enough the sets $\mathcal{G}_{i}^{k}$ are disjoint, this proves that the conditions of Theorem 2 are fulfilled for big $k$. Then by Theorem 2 , we can build a function $u_{k}$ on $\Omega_{k}$ such that $u_{k}$ tends to $-\infty$ (resp. $+\infty$ ) on $\left[Q_{i}^{k}, P_{i+1}\right]$ (resp. on $\left.\left[P_{i}, Q_{i}^{k}\right]\right)$ and $u_{k}\left(P_{0}\right)=0$. Following Remark 3 in section 
3. we shall prove that this sequence $\left(u_{k}\right)$ of solutions of (MSE) has no line of divergence, then the limit $u$ of $\left(u_{k}\right)$ will be our solution. We shall make discussions that are similar to the ones made by H. Jenkins and J. Serrin. We note $\mathrm{d} \Psi_{u_{k}}=\mathrm{d} \Psi_{k}$. We recall that, if $T$ is a segment included in a line of divergence, $\left|\int_{T} \mathrm{~d} \Psi_{k}\right|$ converge to the length of $T$ for a subsequence.

Suppose there exists a line of divergence $L$. We first prove that $L$ can not have an end point in the interior of a $L_{i}^{+}$or a $L_{i}^{-}$. Suppose that $L$ has an end point $D$ in $L_{i}^{-}$(the same argument works for $L_{i}^{+}$). Let $A$ be a point in $L \cap S_{i-1}$, we orient $L$ by $\overrightarrow{A D}$, we suppose that the limit normal along $L$ points on the right-hand side of $L$. We chose a point $B$ of $L_{i}^{-}$on the right-hand side of $D$. Because of the triangle inequality, there exists a point $C$ on $[A, D]$ such that $|A C|+|D B|>|C D|+|B A|$. for $k$ big enough we have $A$ and $C$ in $\Omega_{k}$ we then put $D_{k}=[A, D] \cap\left[P_{i}, Q_{i-1}^{k}\right]$ and $B_{k}=[A, B] \cap\left[P_{i}, Q_{i-1}^{k}\right]$ (see Figure 3 .

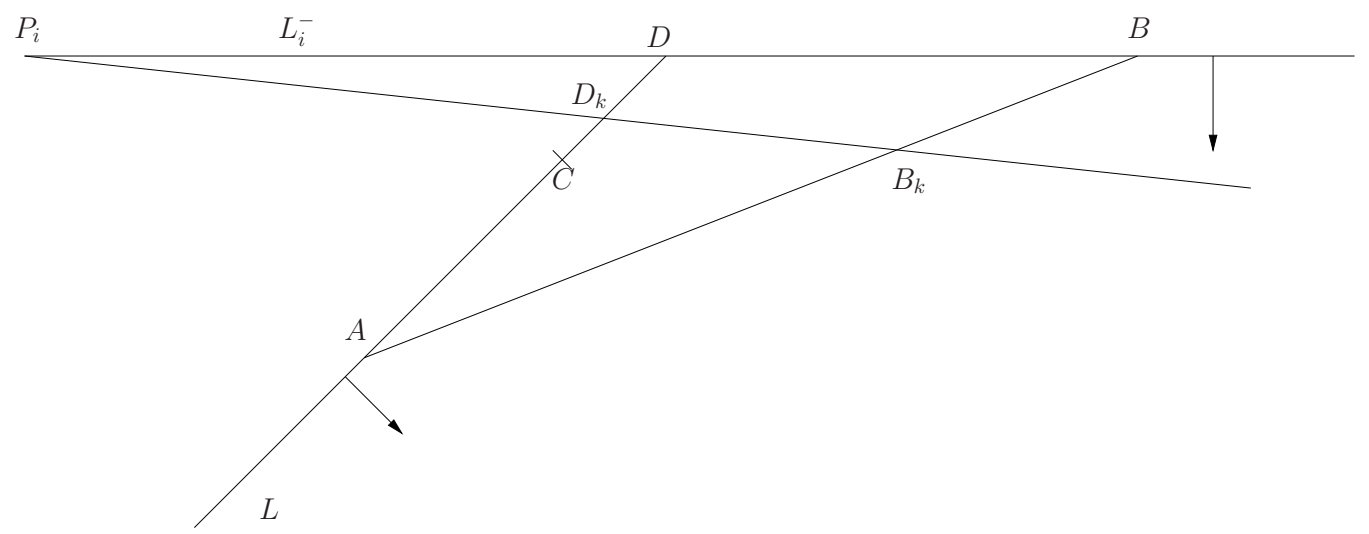

Figure 3:

Let $T_{k}$ be the triangle $A D_{k} B_{k}$ with this orientation. We then have:

$$
\begin{aligned}
0=\int_{T_{k}} \mathrm{~d} \Psi_{k} & \geq \int_{[A, C]} \mathrm{d} \Psi_{k}-\left|C D_{k}\right|+\left|D_{k} B_{k}\right|-\left|B_{k} A\right| \\
& \geq \int_{[A, C]} \mathrm{d} \Psi_{k}-|C D|+\left|D_{k} B_{k}\right|-|B A|
\end{aligned}
$$

But $\left|D_{k} B_{k}\right| \longrightarrow|D B|$ and $\int_{[A, C]} \mathrm{d} \Psi_{k} \longrightarrow|A C|$ for the subsequence that makes $L$ appear; this gives us a contradiction. 
We have now only a finite number of possibilities for a line of divergence. If it has an end point, it must be one $P_{i}$. By construction, we have $\int_{\left[P_{i}, P_{i+1}\right]} \mathrm{d} \Psi_{k}=0$ (because the integral of $\mathrm{d} \Psi_{k}$ along the triangle $P_{i} Q_{i}^{k} P_{i+1}$ is zero and we know $\mathrm{d} \Psi_{k}$ along $\left[P_{i} Q_{i}^{k}\right]$ and $\left[Q_{i}^{k} P_{i+1}\right]$ by Lemma 10 so if $\Gamma$ is a curve joining $P_{i}$ to $P_{j}$ we have $\int_{\Gamma} \mathrm{d} \Psi_{k}=0$. Then, by passing to the limit, if $\Gamma$ is a line of divergence, we obtain $\left|P_{i} P_{j}\right|=0$ which is not possible. This proves that a line of divergence has at most one end point. Suppose that a line of divergence $L$ has no end point, we are in the situation of Figure 4. Let $A$ and $B$ be point on $L$ as in Figure 4 such that $|A B|>\left|P_{i} P_{i+1}\right|$. We note $D$ (resp. $C$ ) the projection of $A$ (resp. $B$ ) on $L_{i+1}^{-}$. For $k$ big enough we note $C_{k}=[B, C] \cap\left[P_{i+1}, Q_{i}^{k}\right]$ and $D_{k}=[A, D] \cap\left[P_{i+1}, Q_{i}^{k}\right]$. We then have:

$$
\begin{aligned}
0=\int_{A B C_{k} D_{k}} \mathrm{~d} \Psi_{k} & \geq \int_{[A, B]} \mathrm{d} \Psi_{k}-\left|B C_{k}\right|+\int_{\left[C_{k}, D_{k}\right]} \mathrm{d} \Psi_{k}-\left|D_{k} A\right| \\
& \geq \int_{[A, B]} \mathrm{d} \Psi_{k}-2\left|P_{i}, P_{i+1}\right|+\left|C_{k} D_{k}\right|
\end{aligned}
$$

We have $\left|C_{k} D_{k}\right| \longrightarrow|C D|=|A B|$ and $\int_{[A, B]} \mathrm{d} \Psi_{k} \longrightarrow|A B|$ for a subsequence, so we get a contradiction and a line of divergence must have one end point.

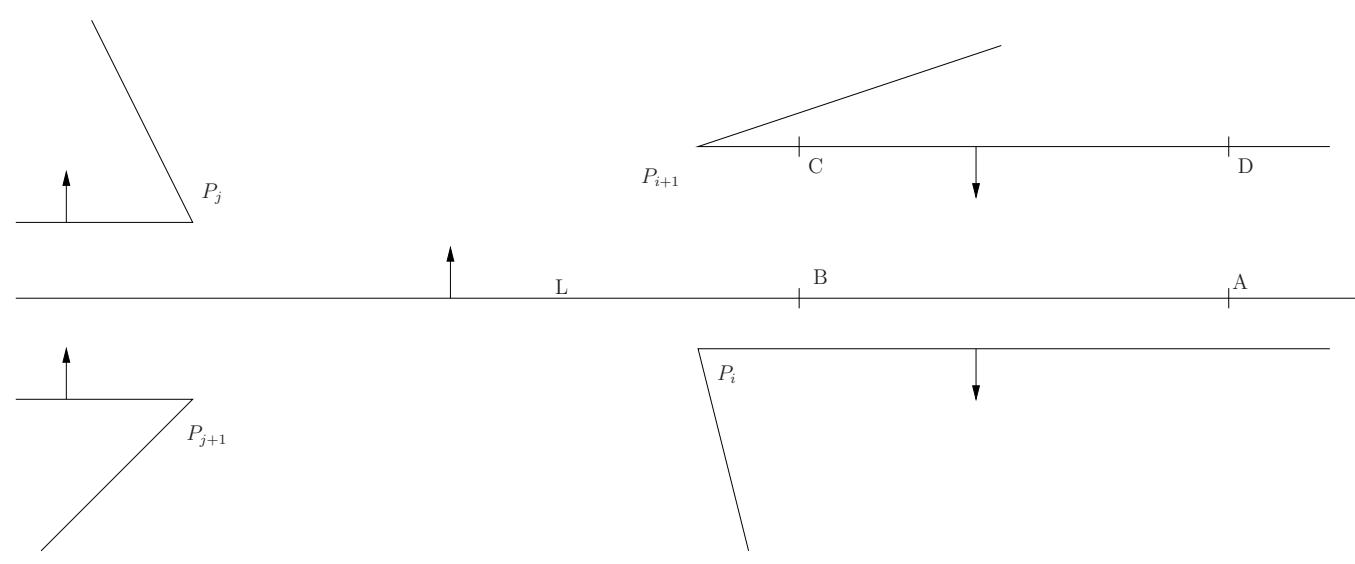

Figure 4:

Let $L$ be a line of divergence, we know that we are in the case where $L$ has $P_{i}$ as end point and goes to infinity in one $S_{j}$. By what we have done just 
above, we have only one possiblity for the limit normal: we are in the same situation as in the semi-strip $S_{j}$ in Figure 4. Then, by changing $L$ if necessary, we can suppose that the part of $L$ in $S_{j}$ is parametrized by $\{A\} \times \mathbb{R}_{+}$with $A \in] P_{j}, P_{j+1}\left[\right.$ and the domain $\widetilde{\Omega}$ parametrized by $\left[A, P_{j+1}\right] \times \mathbb{R}_{+}$is in $\mathcal{B}\left(u_{k}\right)$. Let $\theta$ be an extraction that makes $L$ appear, since $\widetilde{\Omega} \subset \mathcal{B}\left(u_{k}\right)$, there exists an extraction $\theta^{\prime}$ such that $u_{\theta^{\prime}(k)}$ is a subsequence of $u_{\theta(k)}$ and $u_{\theta^{\prime}(k)}-u_{\theta^{\prime}(k)}(K)$ (where $K \in \widetilde{\Omega}$ ) converges to $v$ a solution of (MSE) on $\widetilde{\Omega}$.

We shall now prove that $v$ tends to $-\infty$ on $L_{j+1}^{-}$and $+\infty$ on $L$. Let $B \in\left[A, P_{j+1}\right]$ and $\mathrm{C}$ and $\mathrm{D}$ be the points which are respectively parametrized by $(B, c)$ and $(B, d)(c<d)$. We note:

$$
\begin{gathered}
E \text { the projection of } D \text { on } L_{j+1}^{-} \\
F \text { the projection of } C \text { on } L_{j+1}^{-} \\
G \text { the projection of } D \text { on } L \\
H \text { the projection of } C \text { on } L
\end{gathered}
$$

We note also, when $k$ is big enough, $E_{k}=[D, E] \cap\left[P_{j+1}, Q_{j}^{k}\right]$ and $F_{k}=$ $[C, F] \cap\left[P_{j+1}, Q_{j}^{k}\right]$. Because $\mathrm{d} \Psi_{k}$ is closed we have:

$$
\begin{gathered}
\left|\int_{[C, D]} \mathrm{d} \Psi_{\theta^{\prime}(k)}-\right| F_{\theta^{\prime}(k)} E_{\theta^{\prime}(k)}|| \leq 2\left|B P_{j+1}\right| \\
\left|\int_{[C, D]} \mathrm{d} \Psi_{\theta^{\prime}(k)}-\int_{[H, G]} \mathrm{d} \Psi_{\theta^{\prime}(k)}\right| \leq 2|B A|
\end{gathered}
$$

Thus, in letting $k$ tends to infinity, we obtain:

$$
\begin{gathered}
\left|\int_{[C, D]} \mathrm{d} \Psi_{v}-\right| C D|| \leq 2\left|B P_{j+1}\right| \\
\left|\int_{[C, D]} \mathrm{d} \Psi_{v}-\right| C D|| \leq 2|B A|
\end{gathered}
$$

So we can calculate $\mathrm{d} \Psi_{v}$ on $L$ and $L_{j+1}^{-}$, we remark that $\mathrm{d} \Psi_{v}$ has the same behaviour as if $v$ assumes the boundary values $+\infty$ on $L$ and $-\infty$ on $L_{j+1}^{-}$. We prove that this is, in fact, the case. We consider now two points $A_{1}$ and $A_{2}$ on $L \cap \widetilde{\Omega}$ and two points $A_{3}$ and $A_{4}$ on $L_{j+1}^{-}$. There exists a solution $v^{\prime}$ of (MSE) on the domain bounded by the polygon $A_{1} A_{2} A_{3} A_{4}$ such that $v^{\prime}=v$ on $\left[A_{1}, A_{4}\right]$ and $\left[A_{2}, A_{3}\right], v^{\prime}$ tends to $+\infty$ on $\left[A_{1}, A_{2}\right]$ and tends to $-\infty$ on $\left[A_{3}, A_{4}\right]$. Since we know the value of $\mathrm{d} \Psi_{v}$ on $\left[A_{1}, A_{2}\right]$ and $\left[A_{3}, A_{4}\right]$, the 
uniqueness part of the proof of Theorem 2 proves that $v=v^{\prime}$. We then have proved that $v$ tends to $+\infty$ on $L$ and $-\infty$ on $L_{j+1}^{-}$.

We shall now get a contradiction to the existence of the line of divergence $L$. We have $\int_{\left[A, P_{j+1}\right]} \mathrm{d} \Psi_{v}=\int_{\widetilde{\Omega} \cap I_{j, l}} \mathrm{~d} \Psi_{v}$, then, by (15) and letting $l$ tends to infinity, we get $\int_{\left[A, P_{j+1}\right]} \mathrm{d} \Psi_{v}=0$. If we follow $L$ between $P_{i}$ and $A$ and the segment $\left[A, P_{j+1}\right]$, we get a path joining $P_{i}$ to $P_{j+1}$. Then we have:

$$
0=\int_{\left[P_{i}, A\right]} \mathrm{d} \Psi_{\theta^{\prime}(k)}+\int_{\left[A, P_{j+1}\right]} \mathrm{d} \Psi_{\theta^{\prime}(k)}
$$

Let $k$ tend to infinity, we get $0=\left|P_{i} A\right|+\int_{\left[A, P_{j+1}\right]} \mathrm{d} \Psi_{v}=\left|P_{i} A\right|$; but $P_{i} \notin\left[P_{j}, P_{j+1}\right]$, this is our contradiction.

We then have prove that $\mathcal{B}\left(u_{k}\right)=\Omega(\mathcal{P})$, as $u_{k}\left(P_{0}\right)=0$ for all $k$ there exists a subsequence $u_{k^{\prime}}$ which converges to a solution $u$ of (MSE). The same arguments that we used just above for $v$ prove that $u$ tends to $+\infty$ $($ resp. $-\infty)$ on $L_{i}^{-}\left(\operatorname{resp} . L_{i}^{+}\right)$; we have then established Theorem 6.

We are then able to build the solution to the Plateau problem at infinity. Let $V$ be a polygon and $(\mathcal{P}, \varphi)$ a polygonal disk bounded by $V$. We consider the solution $u$ of the Dirichlet problen given by Theorem [6. We note $P_{1}, \ldots, P_{r}$ the vertices of $V$, we consider $\Psi_{u}$ normalized by $\Psi_{u}\left(P_{1}\right)=0$, from the proof above, we have $\Psi_{u}\left(P_{i}\right)=0$ for all $i$. Then on $L_{i}^{+}$and $L_{i}^{-}$we have $\Psi_{u}(Q)=\left|Q P_{i}\right|$; since $\Psi_{u}$ is 1-Lipschitz continuous, we have $\Psi_{u}(Q) \geq 0$ for all $Q \in S_{i}$. Suppose that $\left\{Q \in \Omega(\mathcal{P}) \mid \Psi_{u}(Q) \leq 0\right\}$ is not reduced to $\left\{P_{1}, \ldots, P_{r}\right\}$ then there exists a point in the interior of $\mathcal{P}$ such that $\Psi_{u}$ is minimal at this point. But $\Psi_{u}$ corresponds to $x_{3}^{*}$, the third coordinate on $M^{*}$ the conjugate surface to the graph of $u$; since $x_{3}^{*}$ is harmonic on $M^{*}$, it can not have a minimum in the interior of $M^{*}$. We then have proved that $\Psi_{u}>0$ in the interior of $\Omega(\mathcal{P})$.

By Theorem 3, the boundary of the graph of $u$ is composed of the $r$ vertical lines over the points $\varphi\left(P_{i}\right)$. Let $M$ be the graph of $u$ with these $r$ vertical lines. We consider $M^{*}$ the conjugate surface to $M$. The boundary of $M^{*}$ is composed of $r$ horizontal planar geodesic curves, since $\Psi_{u}\left(P_{i}\right)=0$ for all $i$ the $r$ curves are all in the plane $\left\{x_{3}=0\right\}$. Finally, we consider $\Sigma$ the union of $M^{*}$ and of its symmetry by $\left\{x_{3}=0\right\}$. The surface $\Sigma$ is a regular minimal surface, it is complete and its flux polygon is $V$ by construction. By construction, we know also that $\Sigma$ is strongly symmetric with respect to $\left\{x_{3}=0\right\}$. 
The last thing we have to prove about $\Sigma$ for being sure that it is the solution of the Plateau problem at infinity is that it has finite total curvature.

We know (see [11]) that there exists a constant $c$ such that if $u$ is a solution of (MSE) on a domain $D$ and $A \in D, M$ is the graph of $u$ and $d$ is the distance along $M$ of the point in $M$ over $A$ to the boundary of $S$ then the curvature $K$ of $M$ at the point over $A$ is bounded by $\frac{c}{d^{2} W^{2}(A)}$.

Let us consider a half-strip $S=[0, a] \times \mathbb{R}_{+}$and $u$ a solution of (MSE) on $S$ such that $u$ takes the value $+\infty$ (resp. $-\infty)$ on $\{0\} \times \mathbb{R}_{+}^{*}\left(\right.$ resp. $\left.\{a\} \times \mathbb{R}_{+}^{*}\right)$. The boundary of the graph of $u$ is over $[0, a] \times\{0\}$. We then have $K(x, y) \leq$ $\frac{c}{x^{2} W^{2}(x, y)}$. We consider the part $S^{\prime} \subset S$ such that $x \geq x_{0}>0$. For a domain $D$ we note $K(D)$ the total curvature of the graph over $D$. We then have:

$$
\begin{aligned}
K\left(S^{\prime}\right) & =\int_{S^{\prime}} K(x, y) W(x, y) \mathrm{d} x \mathrm{~d} y \\
& \leq \int_{S^{\prime}} \frac{c}{x^{2} W(x, y)} \mathrm{d} x \mathrm{~d} y \\
& \leq \int_{x_{0}}^{+\infty} \frac{c a}{x^{2}} \mathrm{~d} x=\frac{c a}{x_{0}}<+\infty
\end{aligned}
$$

We now use arguments that are similar to the first part of the proof of Theorem 3. We consider, for $\alpha \in] 0, \frac{\pi}{2}[$,

$S(\alpha)=\{(x, y) \in S \mid a-y \leq x \tan \alpha\}$. Let us take $\alpha$ such that $\tan \alpha<\frac{1}{8}$, then Lemma 1 of [7] proves that for every $(x, y) \in S(\alpha) q(x, y)<0$. We note $L(\alpha)$ the segment in $S(\alpha)$ such that $a-y=x \tan \alpha$. By Lemma 2, $u$ is lower-bounded by $m_{1}$ on $L(\alpha)$ and upper bounded by $m_{2}$ on the part of $L$ such that $x<x_{0}<a$. We then define $\Theta:(x, y) \mapsto(x, u(x, y))$. $\Theta$ is a diffeomorphism of $S(\alpha)$ into its image $\operatorname{im} \Theta$. We define $\chi=\Theta^{-1}$ then $\chi_{(2)}$ is a solution of MSE on im $\Theta$. We observe that $\chi$ extends smoothly to $\Theta(L)$. We have $\chi_{(2)}(x, z)$ tends to $a$ as $x$ tends to 0 so we can extend $\chi_{(2)}$ by symmetry to $\operatorname{im} \Theta \cup\left\{(x, z) \in \mathbb{R}^{2} \mid(-x, z) \in \operatorname{im} \Theta\right\}$. To compute the total curvature of the graph of $u$ over $D(\alpha) \cap\left\{x<x_{0}\right\}$, we use its parametrization as a graph over $\operatorname{im} \Theta \cap\left\{0 \leq x \leq x_{0}\right\}$ : 


$$
\begin{aligned}
K\left(D(\alpha) \cap\left\{x<x_{0}\right\}\right)= & K\left(\operatorname{im} \Theta \cap\left\{0 \leq x \leq x_{0}\right\}\right) \\
= & \int_{\operatorname{im} \Theta \cap\left\{0 \leq x \leq x_{0}\right\} \cap\left\{z \leq m_{1}-1\right\}} K(x, z) W(x, z) \mathrm{d} x \mathrm{~d} z \\
& +\int_{\operatorname{im} \Theta \cap\left\{0 \leq x \leq x_{0}\right\} \cap\left\{z \geq m_{1}-1\right\}} K(x, z) W(x, z) \mathrm{d} x \mathrm{~d} z \\
\leq & \int_{\operatorname{im} \Theta \cap\left\{0 \leq x \leq x_{0}\right\} \cap\left\{z \leq m_{1}-1\right\}} \frac{c}{\left(z-m_{1}\right)^{2} W(x, z)} \mathrm{d} x \mathrm{~d} z \\
& +\int_{\operatorname{im} \Theta \cap\left\{0 \leq x \leq x_{0}\right\} \cap\left\{z \geq m_{1}-1\right\}} K(x, z) W(x, z) \mathrm{d} x \mathrm{~d} z \\
\leq & \int_{1}^{+\infty} \frac{c x_{0}}{z^{2}} \mathrm{~d} z+C
\end{aligned}
$$

because $\overline{\operatorname{im} \Theta} \cap\left\{0 \leq x \leq x_{0}\right\} \cap\left\{z \geq m_{1}-1\right\}$ is compact

$$
<+\infty
$$

We can do the same work for $\{(x, y) \in S \mid y \leq x \tan \alpha\}$.

We then control the curvature on each semi-strip $S_{i}$. There is a last part in $\Omega(\mathcal{P})$. This part is compact and by Lemma $2 u$ is bounded on this part; besides the graph is regular at the boundary. So the graph above this last part is a compact part of the whole graph then it has finite total curvature. We then have proved that the graph $M$ has finite total curvature. Since $M^{*}$ is isometric to $M$, it has finite total curvature and then $\Sigma$ has finite total curvature because it is twice as many as the one of $M^{*}$.

\section{A The Carathéodory's Theorem}

In this section, we give some explanations on an argument of the proof of Theorem 3 The problem is: when we have a biholomorphic map between two open sets of $\mathbb{C}$, can we extend it to the boundary?

We consider $U$ an open set included in $\mathbb{C}$ and $P$ a point of $\partial U$. We say that $P$ has the property of Schönflies if, for all radii $R$, there exists a radius $r=r(R)$ such that for all two points in $U \cap D(P, r)$ there exists a path in $U \cap D(P, R)$ joining these two points.

We then have the following theorem that we use in our proof.

Theorem 7 (Carathéodory). Let $U$ be a simply connected open set in $\mathbb{C}$ and $V$ an open set of the boundary of $U$. We consider $f: U \longrightarrow D=\{z \in$ 
$\mathbb{C}|| z \mid<1\}$ a biholomorphic map. We suppose that every point of $V$ has the property of Schöflies, then $f$ extends to an homeomorphism from $U \cup V$ into $D \cup C$ where $C \subset \partial D$.

A proof of this theorem can be found in [5]. In our proof, we have to verify the property of Schönflies at the points of a part of the boundary. We know that this part of the boundary is embedded in $\mathbb{C}$ so we can build neigh-

borhoods of every point of the boundary in using $\varepsilon$-tubular neighborhood of the boundary. These neighborhoods prove that we have the property of Schönflies.

\section{References}

[1] P. Collin and R. Krust, Le problème de Dirichlet pour l'équation des surfaces minimales sur des domaines non bornés, Bul. Soc. Math. France. 119 (1991), 443-462.

[2] C. Cosín And A. Ros, A Plateau problem at infinity for properly immersed sufaces with finite total curvature, Indiana Univ. Math. J. 50 (2001),847-879.

[3] R. Courant and D. Hilbert, Methods of Mathematical Physics, Vol. II (Interscience, 1962).

[4] D. Gilbarg and N.S. Trudinger, Elliptic Partial Differential Equations of Second Order, 2nd edit. (Springer-Verlag, 1983).

[5] M. Hervé, Les Fonctions Analytiques (Presses Universitaires de France, 1982).

[6] D. Hoffman and H. Karcher, Complete embedded minimal surfaces of finite total curvature, Geometry V, Encylopaedia Math. Sci. 90 (1997) $5-93$

[7] H. Jenkins and J. Serrin, Variational problems of minimal surface type II, Arch. Rational Mech. Anal. 21 (1966), 321-342.

[8] H. Karcher, Embedded minimal surfaces derived from Scherk's examples, Manuscripta Math. 62 (1988) 83-114

[9] J.C.C. Nitsche, On new results on the theory of minimal surfaces, Bull. Amer. Math. Soc. 71 (1965), 195-270. 
[10] J.C.C. Nitsche, Vorlesung über Minimalflächen, Die Grundlehren der mathematischen Wissenschaften no. 199 (Springer-Verlag, 1975).

[11] R. Osserman, A Survey On Minimal Surfaces, Van Nostrand Math. Studies. 1969. 